\title{
Anonymous Constant-Size Ciphertext HIBE From Asymmetric Pairings
}

\author{
Somindu C. Ramanna and Palash Sarkar \\ Applied Statistics Unit \\ Indian Statistical Institute \\ 203, B.T. Road, Kolkata \\ India 700108. \\ e-mail: \{somindu_r,palash\}@isical.ac.in
}

\begin{abstract}
We present a new hierarchical identity based encryption (HIBE) scheme with constant-size ciphertext that can be implemented using the most efficient bilinear pairings, namely, Type-3 pairings. In addition to being fully secure, our scheme is anonymous. The HIBE is obtained by extending an asymmetric pairing based IBE scheme due to Lewko and Waters. The extension uses the approach of Boneh-Boyen-Goh to obtain constant-size ciphertexts and that of Boyen-Waters for anonymity. Security argument is based on the dual-system technique of Waters. The resulting HIBE is the only known scheme using Type-3 pairings achieving constant-size ciphertext, security against adaptive-identity attacks and anonymity under static assumptions without random oracles.
\end{abstract}

Keywords: identity-based encryption(IBE), constant-size ciphertext hierarchical IBE, asymmetric pairings, dual-system encryption

\section{Introduction}

The notion of identity-based encryption (IBE) was introduced by Shamir [15] and the first IBE schemes appeared later [6,3]. In IBE, a sender encrypts a message using the receiver's identity itself as the public key and a central authority called private key generator (PKG) generates and securely distributes decryption keys corresponding to identities of different users. Hierarchical IBE (HIBE), proposed by $[10,11]$, reduces the workload of the PKG by allowing it to delegate the key generation ability to lower-level entities. As a result, an individual user can conveniently obtain a decryption key from a lower-level entity instead of obtaining it from the PKG.

Type-3 Pairings: Most practical (H)IBE schemes are built using a bilinear pairing which maps $\mathbb{G}_{1} \times \mathbb{G}_{2}$ to $\mathbb{G}_{T}$, where $\mathbb{G}_{1}, \mathbb{G}_{2}$ and $\mathbb{G}_{T}$ are groups of the same order. Well-known examples of such maps arise by suitably choosing $\mathbb{G}_{1}$ and $\mathbb{G}_{2}$ to be groups of elliptic curve points and $\mathbb{G}_{T}$ to be a subgroup of the multiplicative group of a finite field. From an implementation point of view, it is most efficient to use bilinear maps where the (common) group order is prime and it is computationally difficult to find an isomorphism from $\mathbb{G}_{1}$ to $\mathbb{G}_{2}$ or vice versa. Such pairings are called Type-3 pairings $[5,16,9]$. Less efficient alternatives are when $\mathbb{G}_{1}$ and $\mathbb{G}_{2}$ are same (called Type-1 pairings) or when the common group order is a composite number (called composite-order pairings). IBE or HIBE schemes based on Type-3 pairings would have the fastest algorithms and the most compact representations of parameters.

Constant-Size Ciphertext HIBE: In HIBE, identities consist of tuples of varying lengths. Encryption of a message is done for a specified identity tuple. In many HIBE schemes, as the length of the identity tuple increases, so does the length of the resulting ciphertext. Consequently, the bandwidth requirement for communicating the ciphertext also increases.

The solution to this issue is to require that the ciphertext size is independent of the length of the identity tuple. Then, irrespective of the length of the identity tuple, the bandwidth required for 
the ciphertext would be the same. Such a scheme is called a constant-size ciphertext HIBE. The first such HIBE scheme was proposed by Boneh, Boyen and Goh [2]. While the scheme itself is quite elegant, its proof of security was in a very restricted attack model, the so-called selective-identity model. Lewko and Waters [12] provided the first constant-size ciphertext HIBE scheme which is secure against the usual adaptive-identity attacks. The drawback, however, was that the scheme in [12] used pairings on composite order groups and could not be instantiated with the more efficient Type-3 pairings.

In the following, we use the abbreviation CC-HIBE to denote HIBE schemes with constant-size ciphertexts. We clarify that the constant size here only refers to the number of group elements in the ciphertext. The size of representation of the group elements, however, needs to increase if the value of the security parameter increases.

Anonymity: In (H)IBE schemes with anonymity, ciphertexts do not reveal any information about the identity of the recipient. Abdalla et.al. [1] first formalised the notion of anonymity and used it to construct public key encryption with keyword search (PEKS). PEKS enables search on encrypted documents based on some keywords and this capability for search is delegateable. Anonymous HIBE schemes provide means to extend PEKS to more sophisticated primitives such as public key encryption with temporary keyword search (PETKS) and identity-based encryption with keyword search (IBEKS). The first construction of anonymous HIBE without random oracles was given by [4] with security in the selective-id model. Later construction by $[14,7]$ could achieve security in the adaptive-id setting but were based on composite-order pairings. Two other constructions [8, 13] used asymmetric pairings but with security in the selective-id model.

\subsection{Our Contributions}

Our main motivation in this work is to obtain a constant-size ciphertext HIBE which can be implemented using Type-3 pairings. This allows the benefits of having constant-size ciphertexts to be combined with the efficiency benefits of using Type-3 pairings. These efficiency considerations are attained while retaining the usual provable guarantees, namely security against adaptive-identity attacks, use of static hardness assumptions, no degradation of security with increase in the depth of the HIBE and the avoidance of random oracles.

The provable properties are achieved using the extremely useful idea of dual-system encryption introduced by Waters [17]. This technique was used by Lewko and Waters [12] to construct an IBE and a CC-HIBE scheme based on composite-order pairings. The authors in [12] went on to convert their composite-order pairing based IBE scheme to one which can be instantiated using Type-3 pairings. However, no such conversion was done for the HIBE scheme in [12] and the authors do not make any remark on whether this can be done or how difficult it would be to do so.

The starting point of our work are the IBE schemes in [12]. Two IBE schemes are given in [12] where the first one is in the setting of composite order groups and the second one is in the Type-3 setting. The IBE in the composite order setting is not anonymous as has been shown in [14]. On the other hand, the question of anonymity of Type-3 variant has not been addressed by any follow-up work. By LW-IBE we will refer to the IBE in the Type-3 setting appearing in [12]. Two static (though non-standard) computational assumptions (which we denote as LW1 and LW2) are used in [12] to show the security of LW-IBE. The first contribution of the current work is to show that the LW-IBE is anonymous. For this, we need to introduce a new computational assumption which is again static, but, non-standard.

The main contribution of this paper is to extend the LW-IBE to a constant-size ciphertext HIBE. At a very basic level, the idea for obtaining constant-sized ciphertexts is to use the identity 
hashing technique suggested in [2] over existing IBE schemes. We will refer to this as BBG-hash or BBG-extension. Unlike that of [12], we do not take the path of composite-order pairings. Instead, we start with LW-IBE and extend it to a CC-HIBE by plugging in the BBG-hash. One complication in doing so arises - in the dual-system technique, one has to appropriately define semi-functional components which have to be generated by the simulator while handling key extraction queries in the security reduction. Extending the decryption key of LW-IBE to the decryption key of a HIBE in a straightforward manner does not retain the structure required for a dual-system proof. Our way of tackling this is to add additional components to the decryption key. On the face of it, this complicates the key generation and delegation mechanisms. However, somewhat counter-intuitively, adding this extra level of complication allows the security reductions to go through.

An offshoot of adding these extra components is that the scheme becomes anonymous. A technique for obtaining anonymity introduced in [4] and later used by [14] in the context of CC-HIBE, is implicitly used in our construction. Surprisingly, if we try to make the scheme non-anonymous hoping to make it more efficient, the dual system proof will fail. This is because the semi-functional space (for both ciphertexts and keys) is created using some secret random elements which could be embedded in the key. In the HIBE scheme, in order to facilitate re-randomisation during delegation all these elements may have to be revealed in the public parameters! This in turn affects the dual-system proof. A natural question that follows is whether we can prove anonymity for the scheme. The answer is positive and the scheme is anonymous.

The computational assumptions required to obtain security are those used in [12] along with the new assumption required to show that the LW-IBE is anonymous. The last assumption is used to prove the anonymity of the HIBE scheme.

\section{Preliminaries}

\subsection{Notation}

For a set $\mathcal{X}$, the notation $x_{1}, \ldots, x_{k} \in_{\mathrm{R}} \mathcal{X}$ (or $x_{1}, \ldots, x_{k} \stackrel{\mathrm{R}}{\longleftarrow} \mathcal{X}$ ) indicates that $x_{1}, \ldots, x_{k}$ are elements of $\mathcal{X}$ chosen independently at random according to some distribution $\mathrm{R}$. We use two notations interchangeably. The uniform distribution is denoted by U. For a (probabilistic) algorithm $\mathcal{A}, x \longleftarrow \mathcal{A}(\cdot)$ means that $x$ is chosen according to the output distribution of $\mathcal{A}$ (which of course may be determined by its input). For two integers $a<b$, the notation $[a, b]$ represents the set $\{x \in \mathbb{Z}: a \leq x \leq b\}$. Let $\mathbb{G}$ be a finite cyclic group and $\mathbb{G}^{\times}$denote the set of generators of $\mathbb{G}$. Fix a generator $P_{1} \in \mathbb{G}^{\times}$. Discrete logarithm of an element $Q \in \mathbb{G}$ to base $P_{1}$ is written as $\operatorname{dlog}_{P_{1}} Q$.

\subsection{Bilinear pairings}

A bilinear pairing is given by a 7 -tuple $\mathcal{G}=\left(p, \mathbb{G}_{1}, \mathbb{G}_{2}, \mathbb{G}_{T}, e, P_{1}, P_{2}\right)$ where $\mathbb{G}_{1}=\left\langle P_{1}\right\rangle, \mathbb{G}_{2}=\left\langle P_{2}\right\rangle$ are additive groups and $\mathbb{G}_{T}$ is a multiplicative group, all having the same order $p$ and $e: \mathbb{G}_{1} \times \mathbb{G}_{2} \rightarrow \mathbb{G}_{T}$ is a map with the following properties.

1. Bilinear: For $P_{1}, Q_{1} \in \mathbb{G}_{1}$ and $P_{2}, Q_{2} \in \mathbb{G}_{2}$, the following holds: $e\left(P_{1}, P_{2}+Q_{2}\right)=e\left(P_{1}, P_{2}\right) e\left(P_{1}, Q_{2}\right)$ and $e\left(P_{1}+Q_{1}, P_{2}\right)=e\left(P_{1}, P_{2}\right) e\left(Q_{1}, P_{2}\right)$.

2. Non-degenerate: If $e\left(P_{1}, P_{2}\right)=1_{T}$, the identity element of $\mathbb{G}_{T}$, then either $P_{1}$ is the identity of $\mathbb{G}_{1}$ or $P_{2}$ is the identity of $\mathbb{G}_{2}$.

3. Efficiently computable: The function $e$ should be efficiently computable.

Three main types of pairings have been identified in the literature $[16,9]$. 
Type-1 In this type, the groups $\mathbb{G}_{1}$ and $\mathbb{G}_{2}$ are the same.

Type-2 $\mathbb{G}_{1} \neq \mathbb{G}_{2}$ and an efficiently computable isomorphism $\psi: \mathbb{G}_{2} \rightarrow \mathbb{G}_{1}$ is known.

Type-3 Here, $\mathbb{G}_{1} \neq \mathbb{G}_{2}$ and no efficiently computable isomorphisms between $\mathbb{G}_{1}$ and $\mathbb{G}_{2}$ are known.

It has been reported $[5,16,9]$ that from an implementation point of view, Type-3 pairings are the fastest to compute and further provide the most compact description of group elements. So, building functionalities which can be instantiated with such pairings is of practical interest. This work is entirely based on Type-3 pairings.

We introduce some notation: fix $P_{1} \in \mathbb{G}_{1}^{\times}$and $P_{2} \in \mathbb{G}_{2}^{\times}$; for elements $R_{1} \in \mathbb{G}_{1}$ and $R_{2} \in \mathbb{G}_{2}$, the notation $R_{1} \sim R_{2}$ indicates that $\operatorname{dlog}_{P_{1}} R_{1}=\operatorname{dlog}_{P_{2}} R_{2}$.

\subsection{Complexity Assumptions}

Here, we define certain hardness assumptions in Type-3 setting that are needed for the security reductions. In all the assumptions stated below, $\mathcal{G}=\left(p, \mathbb{G}_{1}, \mathbb{G}_{2}, \mathbb{G}_{T}, e, P_{1}, P_{2}\right)$ is an asymmetric pairing and $\mathscr{A}$ is a probabilistic polynomial time $(\mathrm{PPT})$ algorithm $\mathscr{A}$ that outputs 0 or 1 .

Assumption LW1 [12]. Define a distribution $\mathcal{D}$ as follows: $F_{1} \stackrel{\mathrm{U}}{\longleftarrow} \mathbb{G}_{1}^{\times} ; F_{2} \stackrel{\mathrm{U}}{\longleftarrow} \mathbb{G}_{2}^{\times}, a, b, s \stackrel{\mathrm{U}}{\longleftarrow} \mathbb{Z}_{p}$, $Y_{1} \stackrel{\mathrm{U}}{\longleftarrow} \mathbb{G}_{1} ;$

$$
\mathcal{D}=\left(\mathcal{G}, F_{1}, b s F_{1}, s F_{1}, a F_{1}, a b^{2} F_{1}, b F_{1}, b^{2} F_{1}, a s F_{1}, b^{2} s F_{1}, b^{3} F_{1}, b^{3} s F_{1}, F_{2}, b F_{2}\right) .
$$

The advantage of $\mathscr{A}$ in solving the LW1 problem is given by

$$
\operatorname{Adv}_{\mathcal{G}}^{\mathrm{LW} 1}(\mathscr{A})=\left|\operatorname{Pr}\left[\mathscr{A}\left(\mathcal{D}, a b^{2} s F_{1}\right)=1\right]-\operatorname{Pr}\left[\mathscr{A}\left(\mathcal{D}, Y_{1}\right)=1\right]\right| .
$$

The $(\varepsilon, t)$-LW1 assumption holds in $\mathcal{G}$ if for any adversary $\mathscr{A}$ running in time at most $t$, $\operatorname{Adv}_{\mathcal{G}}^{\mathrm{LW} 1}(\mathscr{A}) \leq \varepsilon$

Assumption LW2 [12]. Let distribution $\mathcal{D}$ be defined as follows: $F_{1} \stackrel{\mathrm{U}}{\longleftarrow} \mathbb{G}_{1}^{\times} ; F_{2} \stackrel{\mathrm{U}}{\longleftarrow} \mathbb{G}_{2}^{\times}$, $d, b, c, x \stackrel{\mathrm{U}}{\longleftarrow} \mathbb{Z}_{p}, Y_{2} \stackrel{\mathrm{U}}{\longleftarrow} \mathbb{G}_{2} ;$

$$
\mathcal{D}=\left(\mathcal{G}, F_{1}, d F_{1}, d^{2} F_{1}, b x F_{1}, d b x F_{1}, d^{2} x F_{1}, F_{2}, d F_{2}, b F_{2}, c F_{2}\right) .
$$

$\mathscr{A}$ 's advantage in solving the LW2 problem is given by

$$
\operatorname{Adv}_{\mathcal{G}}^{\mathrm{LW} 2}(\mathscr{A})=\left|\operatorname{Pr}\left[\mathscr{A}\left(\mathcal{D}, b c F_{2}\right)=1\right]-\operatorname{Pr}\left[\mathscr{A}\left(\mathcal{D}, Y_{2}\right)=1\right]\right| .
$$

Essentially, the problem is to decide whether $Z_{2}=b c F_{2}$ or $Z_{2} \in \in_{\mathrm{U}} \mathbb{G}_{2}$ given $\left(\mathcal{D}, Z_{2}\right)$. The $(\varepsilon, t)$-LW1 assumption holds in $\mathcal{G}$ if for any adversary $\mathscr{A}$ running in time at most $t$, $\operatorname{Adv}_{\mathcal{G}}^{\mathrm{LW} 1}(\mathscr{A}) \leq \varepsilon$. The $(\varepsilon, t)$-LW2 assumption is that, for any $t$-time algorithm $\mathscr{A}, \operatorname{Adv}_{\mathcal{G}}^{\mathrm{LW} 2}(\mathscr{A}) \leq \varepsilon$.

Decisional Bilinear Diffie-Hellman in Type-3 pairings (DBDH-3) [5]. Let $F_{1} \longleftarrow$ $\mathbb{G}_{1}^{\times}, \quad F_{2} \longleftarrow \mathbb{G}_{2}^{\times}, x, y, z \stackrel{\mathrm{U}}{\longleftarrow} \mathbb{Z}_{p}$ and $Y_{T} \stackrel{\mathrm{U}}{\longleftarrow} \mathbb{G}_{T}$. Denote by $\mathcal{D}$, the distribution $\left(\mathcal{G}, F_{1}, x F_{1}, y F_{1}, z F_{1}, F_{2}, x F_{2}, y F_{2}\right)$. Define $\mathscr{A}$ 's advantage in solving the DBDH-3 problem as follows.

$$
\operatorname{Adv}_{\mathcal{G}}^{\mathrm{DBDH}-3}(\mathscr{A})=\left|\operatorname{Pr}\left[\mathscr{A}\left(\mathcal{D}, e\left(F_{1}, F_{2}\right)^{x y z}\right)=1\right]-\operatorname{Pr}\left[\mathscr{A}\left(\mathcal{D}, Y_{T}\right)=1\right]\right| .
$$

We say that the $(\varepsilon, t)$-DBDH-3 assumption holds in $\mathcal{G}$ if $\operatorname{Adv}_{\mathcal{G}}^{\mathrm{DBDH}-3}(\mathscr{A}) \leq \varepsilon$ for every algorithm $\mathscr{A}$ running in time at most $t$. 
Assumption A1. Let $F_{1} \stackrel{\mathrm{U}}{\longleftarrow} \mathbb{G}_{1}^{\times}, F_{2} \stackrel{\mathrm{U}}{\longleftarrow} \mathbb{G}_{2}^{\times}, a, z, d, s, x \stackrel{\mathrm{U}}{\longleftarrow} \mathbb{Z}_{p}$ and

$$
\mathcal{D}=\left(\mathcal{G}, F_{1}, z F_{1}, d z F_{1}, a z F_{1}, a d z F_{1}, s z F_{1}, F_{2}, z F_{2}, a F_{2}, x F_{2},(d z-a x) F_{2}\right) .
$$

The A1 problem is to decide, given $\left(\mathcal{D}, Y_{1}\right)$, whether $Y_{1}=s d z F_{1}$ or $Y_{1} \in_{\mathrm{U}} \mathbb{G}_{1}$. Advantage of algorithm $\mathscr{A}$ in solving $\mathrm{A} 1$ is defined as

$$
\operatorname{Adv}_{\mathcal{G}}^{\mathrm{A} 1}(\mathscr{A})=\left|\operatorname{Pr}\left[\mathscr{A}\left(\mathcal{D}, s d z F_{1}\right)=1\right]-\operatorname{Pr}\left[\mathscr{A}\left(\mathcal{D}, Y_{1}\right)=1\right]\right|
$$

where $Y_{1} \in \in_{\mathrm{U}} \mathbb{G}_{1}$. The $(\varepsilon, t)$-A1 assumption is that any $t$-time algorithm $\mathscr{A} \operatorname{has} \operatorname{Adv}_{\mathcal{G}}^{\mathrm{A} 1}(\mathscr{A}) \leq \varepsilon$.

Discussion. We introduce assumption A1 to show anonymity of LW-IBE as well as our HIBE scheme. The challenge in $\mathrm{A} 1$ is an element $Z_{1} \in \mathbb{G}_{1}$; the task is to decide whether $Z_{1}=s d z F_{1}$ or random. Suppose we can successfully create $e\left(F_{1}, F_{2}\right)^{s d z \delta}$ (for some $\delta$ such that $\delta F_{2}$ is given in the instance) using elements in the instance, then the problem becomes easy to solve - just check for equality with $e\left(Z_{1}, \delta F_{2}\right)$. If they are equal then $Z_{1}$ is real; otherwise $Z_{1}$ is random. Since $s$ and $d$ appear in separate elements in $\mathbb{G}_{1}$, the only possible way is to compute $e\left(Z_{1}, z F_{2}\right)$ and compare it to $e\left((d z-a x) F_{2}, s z F_{1}\right)$ after cancelling out $e\left(F_{1}, F_{2}\right)^{a x s z}$. But this extra term cannot be cancelled since $a$ and $x$ appear in separate elements of $\mathbb{G}_{2}$. So our assumption is meaningful and there does not seem to be any way of efficiently solving A1. Let DDH1 (resp. DDH2) be the decision DiffieHellman assumption in group $\mathbb{G}_{1}$ (resp. $\mathbb{G}_{2}$ ). It is well-known that in Type-3 setting these problems are computationally hard.

The problem LW1 contains an embedded instance of DDH1. The elements $s F_{1}$ and $a b^{2} F_{1}$ are provided in the instance and it is required to determine whether $Y_{1}$ equals $a b^{2} s F_{1}$ or $Y_{1}$ is random. Similarly, LW2 contains an embedded instance of DDH2: the elements $b F_{2}$ and $c F_{2}$ are provided in the instance and it is required to determine whether $Y_{2}$ equals $b c F_{2}$ or $Y_{2}$ is random. As a result, an algorithm to solve DDH1 (resp. LW1) implies an algorithm to solve LW1 (resp. LW2) so that we can say that LW1 (resp. LW2) is no harder than DDH1 (resp. DDH2). The other direction, however, is not clear and it is due to this reason that the assumptions are considered non-standard.

In contrast to the above, the problem $\mathrm{A} 1$ on the other hand, does not contain an embedded instance of DDH1. In this problem, it is required to determine whether $Y_{1}=s d z F_{1}$ or $Y_{1}$ is random. The instance contains $z F_{1}$, but, not $s d F_{1} ; d z F_{1}$ but, not $s F_{1} ; s z F_{1}$ but, not $d F_{1}$ and so on. There does not seem to be any way to use a DDH1 solver to solve A1. So, it does not seem possible to reduce DDH1 to $\mathrm{A} 1$ or vice-versa.

\subsection{Hierarchical Identity-Based Encryption}

A HIBE scheme consists of five probabilistic polynomial time (in the security parameter) algorithms - Setup, Encrypt, KeyGen, Delegate and Decrypt.

- Setup: based on an input security parameter $\kappa$, generates and outputs the public parameters $\mathcal{P P}$ and the master secret $\mathcal{M S K}$.

- KeyGen: inputs an identity vector id and master secret $\mathcal{M S K}$ and outputs the secret key $\mathcal{S} \mathcal{K}_{\text {id }}$ corresponding to id.

- Encrypt: inputs an identity id, a message $M$ and returns a ciphertext $\mathcal{C}$.

- Delegate: takes as input a depth $\ell$ identity vector $\mathbf{i d}=\left(\mathrm{id}_{1}, \ldots, \mathrm{id}_{\ell}\right)$, a secret key $\mathcal{S} \mathcal{K}_{\mathbf{i d}}$ and an identity $\mathrm{id}_{\ell+1}$; returns a secret key for the identity vector $\left(\mathrm{id}_{1}, \ldots, \mathrm{id}_{\ell+1}\right)$.

- Decrypt: inputs a ciphertext $\mathcal{C}$, an identity vector id, secret key $\mathcal{S} \mathcal{K}_{\mathbf{i d}}$ and returns either the corresponding message $M$ or $\perp$ indicating failure. 


\subsection{Anonymous CPA-Secure HIBE}

The following security game captures both anonymity and security against a chosen plaintext attack for a HIBE scheme and will be called ano-ind-cpa.

Setup: The challenger runs the Setup algorithm of the HIBE and gives the public parameters to $\mathscr{A}$.

Phase 1: $\mathscr{A}$ makes a number of key extraction queries adaptively. For a query on an identity vector id, the challenger responds with a key $\mathcal{S} \mathcal{K}_{\text {id }}$.

Challenge: $\mathscr{A}$ provides two message-identity pairs $\left(M_{0}, \widehat{\mathbf{i d}}_{0}\right)$ and $\left(M_{1}, \widehat{\mathbf{i d}}_{1}\right)$ as challenge with the restriction that neither $\widehat{\mathbf{i d}}_{0}, \widehat{\mathbf{i d}}_{1}$ nor any of their prefixes should have been queried in Phase $\mathbf{1}$. The challenger then chooses a bit $\beta$ uniformly at random from $\{0,1\}$ and returns an encryption $\widehat{\mathcal{C}}$ of $M_{\beta}$ under the identity $\widehat{\mathbf{i d}}_{\beta}$ to $\mathscr{A}$.

Phase 2: $\mathscr{A}$ issues more key extraction queries as in Phase $\mathbf{1}$ with the restriction that no queried identity id is a prefix of $\widehat{\mathbf{i d}}_{\beta}$.

Guess: $\mathscr{A}$ outputs a bit $\beta^{\prime}$.

If $\beta=\beta^{\prime}$, then $\mathscr{A}$ wins the game. The advantage of $\mathscr{A}$ in breaking the security of the HIBE scheme in the game ano-ind-cpa given by

$$
\operatorname{Adv}_{\mathrm{HIBE}}^{\text {ano-ind-cpa }}(\mathscr{A})=\left|\operatorname{Pr}\left[\beta=\beta^{\prime}\right]-\frac{1}{2}\right| .
$$

The HIBE scheme is said to be $(\varepsilon, t, q)$-ANO-IND-ID-CPA secure if every $t$-time adversary making at most $q$ queries has $\operatorname{Adv}_{\text {HIBE }}^{\text {ano-ind-cpa }}(\mathscr{A}) \leq \varepsilon$.

\section{Lewko-Waters IBE}

This section reviews the asymmetric pairing-based IBE construction of Lewko-Waters [12]. The description in [12] consists of the usual ciphertexts and keys as well as the so-called semi-functional ciphertexts and keys. The semi-functional versions are obtained by modifying the normal versions. We use a compact notation to denote normal and semi-functional ciphertexts and keys. The group elements shown in curly brackets \{\} are the semi-functional components. To get the scheme itself, these components should be ignored.

Let $\mathcal{G}=\left(p, \mathbb{G}_{1}, \mathbb{G}_{2}, \mathbb{G}_{T}, e, P_{1}, P_{2}\right)$ be an asymmetric pairing. Pick $Q_{1}, U_{1} \in \mathbb{U}_{\mathrm{U}} \mathbb{G}_{1}$ and $Q_{2}, U_{2} \in \mathbb{G}_{2}$ be such that $Q_{2} \sim Q_{1}$ and $U_{2} \sim U_{1}$. Choose $F_{2} \stackrel{\mathrm{U}}{\longleftarrow} \mathbb{G}_{2}^{\times}, a, v, v^{\prime} \stackrel{\mathrm{U}}{\longleftarrow} \mathbb{Z}_{p}$ and define $V_{2}=v F_{2}, V_{2}^{\prime}=$ $v^{\prime} F_{2}$. Let $\tau=v+a v^{\prime}$ so that $\tau F_{2}=V_{2}+a V_{2}^{\prime}$. Identities are elements of $\mathbb{Z}_{p}$. The public parameters and master secret are given by

$\mathcal{P P} \quad:\left(P_{1}, a P_{1}, \tau P_{1}, Q_{1}, a Q_{1}, \tau Q_{1}, U_{1}, a U_{1}, \tau U_{1}, e\left(P_{1}, P_{2}\right)^{\alpha}\right)$

$\mathcal{M S K}:\left(\alpha P_{2}, P_{2}, V_{2}, V_{2}^{\prime}, Q_{2}, U_{2}, F_{2}\right)$.

The randomisers for the ciphertext and key are $s$ and $w, r_{1}, r_{2}$ respectively. These are elements of $\mathbb{Z}_{p}$. For the semi-functional components, $\mu, \sigma$ and $\gamma, \pi$ are chosen at random from $\mathbb{Z}_{p}$. Elements $V_{1}^{\prime}, F_{1} \in \mathbb{G}_{1}$ are such that $V_{1}^{\prime} \sim V_{2}^{\prime}$ and $F_{1} \sim F_{2}$.

\section{Ciphertext:}

$$
\begin{aligned}
& C_{0}=M \times e\left(P_{1}, P_{2}\right)^{\alpha s} \\
& C_{1,1}=s\left(\mathrm{id} Q_{1}+U_{1}\right), C_{1,2}=a s\left(\mathrm{id} Q_{1}+U_{1}\right)\left\{+\mu \sigma F_{1}\right\}, C_{1,3}=-\tau s\left(\mathrm{id} Q_{1}+U_{1}\right)\left\{-\mu \sigma V_{1}^{\prime}\right\} \\
& C_{2,1}=s P_{1}, C_{2,2}=a s P_{1}\left\{+\mu F_{1}\right\}, C_{2,3}=-\tau s P_{1}\left\{-\mu V_{1}^{\prime}\right\}
\end{aligned}
$$


Key:

$$
\begin{aligned}
& K_{1,1}=w P_{2}+r_{1} V_{2}\left\{-a \gamma F_{2}\right\}, K_{1,2}=r_{1} V_{2}^{\prime}\left\{+\gamma F_{2}\right\}, K_{1,3}=r_{1} F_{2} \\
& K_{2,1}=\alpha P_{2}+w\left(\operatorname{id} Q_{2}+U_{2}\right)+r_{1} V_{2}\left\{-a \gamma \pi F_{2}\right\}, K_{2,2}=r_{2} V_{2}^{\prime}\left\{+\gamma \pi F_{2}\right\}, K_{2,3}=r_{2} F_{2}
\end{aligned}
$$

Lewko and Waters show that this scheme is adaptively secure without random oracles under three non-standard but static assumptions - LW1, LW2 and DBDH-3. Since the elements $Q_{2}, U_{2}$ are in the master secret there seems to be no way to check whether a given ciphertext is encrypted to a particular identity or not. In other words, this scheme is anonymous. We provide a proof in the ANO-IND-ID-CPA model which encompasses both CPA-security and anonymity.

Theorem 1. If the $\left(\varepsilon_{\mathrm{LW} 1}, t^{\prime}\right)$-LW1, $\left(\varepsilon_{\mathrm{LW} 2}, t^{\prime}\right)$-LW2, $\left(\varepsilon_{\mathrm{DBDH}-3}, t^{\prime}\right)$-DBDH-3 and $\left(\varepsilon_{\mathrm{A} 1}, t^{\prime}\right)-\mathrm{A} 1$ assumptions hold, then LW-IBE is $(\varepsilon, t, q)$-ANO-IND-ID-CPA secure where

$$
\varepsilon \leq \varepsilon_{\mathrm{LW} 1}+q \varepsilon_{\mathrm{LW} 2}+\varepsilon_{\mathrm{DBDH}-3}+\varepsilon_{\mathrm{A} 1}
$$

and $t=t^{\prime}-O(q \rho)$, where $\rho$ is an upper bound on the time required for one scalar multiplication in $\mathbb{G}_{1}$ or $\mathbb{G}_{2}$.

Proof. Let $\mathscr{A}$ be any $t$-time adversary against LW-IBE in the ano-ind-cpa. The proof follows a hybrid argument over a sequence of $q+4$ games $\mathrm{Game}_{\text {real }}, \mathrm{Game}_{0}, \mathrm{Game}_{1}, \ldots, \mathrm{Game}_{q}, \mathrm{Game}_{M-\text { rand }}$, Game ${ }_{\text {final }}$ - between $\mathscr{A}$ and a simulator $\mathscr{B}$, where the games are defined as follows.

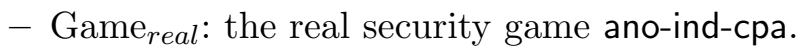

- Game ${ }_{0}$ : challenge ciphertext is semi-functional.

- Game $_{k}(1 \leq k \leq q)$ : first $k$ keys returned to the adversary are semi-functional and the rest are normal.

- Game ${ }_{M-r a n d}$ : the challenge ciphertext encrypts a random message under one of the challenge identities.

- Game $_{\text {final }}$ : both message and challenge identity are random in the challenge ciphertext.

Let $X_{\text {real }}, X_{k}, X_{M \text {-rand }}$ and $X_{\text {final }}$ denote the events that the adversary wins in Game real $_{\text {, }}$, Game $_{k}$, Game $_{M-\text { rand }}$ and Game ${ }_{\text {final }}$ for $0 \leq k \leq q$ respectively. Note that, in Game final $_{\text {, the }}$ challenge ciphertext is an encryption of a random message under a random identity vector. Hence $\beta$ is statistically hidden from the adversary's view implying that $\operatorname{Pr}\left[X_{\text {final }}\right]=1 / 2$. From [12], we know that $\left|\operatorname{Pr}\left[X_{\text {real }}\right]-\operatorname{Pr}\left[X_{0}\right]\right| \leq \varepsilon_{\mathrm{LW} 1},\left|\operatorname{Pr}\left[X_{k-1}\right]-\operatorname{Pr}\left[X_{k}\right]\right| \leq \varepsilon_{\mathrm{LW} 2}$ and $\left|\operatorname{Pr}\left[X_{q}\right]-\operatorname{Pr}\left[X_{M \text {-rand }}\right]\right| \leq \varepsilon_{\mathrm{DBDH}-3}$.

We now show that $\operatorname{Pr}\left[X_{M \text {-rand }}\right]-\operatorname{Pr}\left[X_{\text {final }}\right] \leq \varepsilon_{\mathrm{A} 1}$. Consider a simulator $\mathscr{B}$ playing the game ano-ind-cpa with $\mathscr{A}$. At this stage all keys are semi-functional and the message encrypted in the challenge ciphertext is random. Let $\left(\mathcal{G}, F_{1}, z F_{1}, d z F_{1}, a z F_{1}, a d z F_{1}, s z F_{1}, F_{2}, z F_{2}, a F_{2}, x F_{2},(d z-\right.$ $\left.a x) F_{2}, Z_{1}\right)$ be the instance of $\mathrm{A} 1$ provided to $\mathscr{B}$. Let $Z_{1}=c \cdot s d z F_{1} . \mathscr{B}$ has to determine whether $c=1$ or $c \in \in_{\mathrm{U}} \mathbb{Z}_{p}$. The game is simulated as follows.

Set-Up: Pick $\alpha, v, v^{\prime}, y, u \stackrel{\mathrm{U}}{\longleftarrow} \mathbb{Z}_{p}$ and set the parameters as

$$
\begin{aligned}
& P_{1}=z F_{1}, V_{2}=v F_{2}, V_{2}^{\prime}=v^{\prime} F_{2}, Q_{1}=y\left(d z F_{1}\right), U_{1}=u\left(d z F_{1}\right), \\
& a P_{1}=a z P_{1}, a Q_{1}=y\left(a d z F_{1}\right), a U_{1}=u\left(a d z F_{1}\right)
\end{aligned}
$$

and similarly the elements $\tau P_{1}, \tau Q_{1}$ and $\tau U_{1}$. Compute $e\left(P_{1}, P_{2}\right)^{\alpha}=e\left(z F_{1}, z F_{2}\right)^{\alpha}$. $\mathscr{B}$ returns $\mathcal{P P}$ to $\mathscr{A} . \mathscr{B}$ knows $P_{2}=z F_{2}$ and $\alpha$ but not $Q_{2}$ and $U_{2}$.

Key Extraction Phases 1 and 2: $\mathscr{B}$ picks $w, r_{1}, r_{2} \stackrel{\mathrm{U}}{\longleftarrow} \mathbb{Z}_{p}, \gamma \stackrel{\mathrm{U}}{\longleftarrow} \mathbb{Z}_{p}^{\times}$and $\pi^{\prime} \stackrel{\mathrm{U}}{\longleftarrow} \mathbb{Z}_{p}$. It then computes the key for the $k$-th identity $\mathrm{id}_{k}$ as follows. 


$$
\begin{aligned}
& K_{1,1}=w\left(z F_{2}\right)+r_{1} V_{2}-\gamma a F_{2}, K_{1,2}=r_{1} V_{2}^{\prime}+\gamma F_{2}, K_{1,3}=r_{1} F_{2} \\
& K_{2,1}=\alpha z F_{2}+w\left(y \mathrm{yd}_{k}+u\right)(d z-a x) F_{2}+r_{2} V_{2}-\gamma \pi^{\prime}\left(a F_{2}\right), \\
& K_{2,2}=r_{2} V_{2}^{\prime}+w\left(y \mathrm{id}_{k}+u\right) x F_{2}+\gamma \pi^{\prime} F_{2}, K_{2,3}=r_{2} F_{2},
\end{aligned}
$$

setting $\pi=\pi^{\prime}+\gamma^{-1} w\left(y\right.$ id $\left._{k}+u\right) x$. Since $\pi$ additively randomised it has the correct distribution in $\mathscr{A}$ 's view. $\mathscr{B}$ returns $\mathcal{S} \mathcal{K}_{\text {id }_{k}}=\left(\left(K_{1, i}, K_{2, i}\right)_{i=1,2,3}\right)$ to $\mathscr{A}$. The following calculation shows that $K_{2,1}$ and $K_{2,2}$ are well-formed.

The following calculation shows that $K_{2,1}$ and $K_{2,2}$ are well-formed.

$$
\begin{aligned}
K_{2,1} & =\alpha z F_{2}+w\left(y \operatorname{id}_{i}+u\right)(d z-a x) F_{2}+r_{2} V_{2}-\gamma \pi^{\prime}\left(a F_{2}\right) \\
& =\alpha P_{2}+w\left(y \operatorname{id}_{i}+u\right)(d z-a x) F_{2}+r_{2} V_{2}-\gamma\left(\pi-\gamma^{-1} w\left(y \operatorname{id}_{i}+u\right) x\right)\left(a F_{2}\right) \\
& =\alpha P_{2}+w\left(y \operatorname{id}_{i}+u\right) d z F_{2}-w\left(y \operatorname{id}_{i}+u\right) a x F_{2}+r_{2} V_{2}-a \gamma \pi F_{2}+w\left(y \operatorname{id}_{i}+u\right)\left(a x F_{2}\right) \\
& =\alpha P_{2}+w\left(\operatorname{id}_{i} Q_{2}+U_{2}\right)+r_{2} V_{2}-a \gamma \pi F_{2} \\
K_{2,2} & =r_{2} V_{2}^{\prime}+w\left(y \operatorname{id}_{i}+u\right) x F_{2}+\gamma \pi^{\prime} F_{2} \\
& =r_{2} V_{2}^{\prime}+w\left(y \operatorname{id}_{i}+u\right)\left(x F_{2}\right)+\gamma\left(\pi-\gamma_{1}^{-1} w\left(y \operatorname{id}_{i}+u\right) x\right) F_{2} \\
& =r_{2} V_{2}^{\prime}+w\left(y \operatorname{id}_{i}+u\right)\left(x F_{2}\right)+\gamma \pi F_{2}-w\left(y \operatorname{id}_{i}+u\right) x F_{2} \\
& =r_{2} V_{2}^{\prime}+\gamma \pi F_{2}
\end{aligned}
$$

Challenge: $\mathscr{B}$ receives two pairs of messages and identities $\left(M_{0}, \widehat{\mathrm{id}}_{0}\right)$ and $\left(M_{1}, \widehat{\mathrm{id}}_{1}\right)$ from $\mathscr{A}$. It chooses $\beta \stackrel{\mathrm{U}}{\longleftarrow}\{0,1\}$ and $a^{\prime}, \xi \stackrel{\mathrm{U}}{\longleftarrow} \mathbb{Z}_{p}$ at random and generates a semi-functional challenge ciphertext as follows.

$$
\begin{aligned}
& C_{0} \stackrel{\mathrm{U}}{\longleftarrow} \mathbb{G}_{T} \\
& C_{1,1}=\left(\widehat{y i d}_{\beta}+u\right) Z_{1}, C_{1,2}=a^{\prime}\left(\widehat{\operatorname{id}}_{\beta}+u\right) Z_{1}+\xi F_{1}, \\
& \left.C_{1,3}=-v\left(y \widehat{\mathrm{id}}_{\beta}+u\right)\right) Z_{1}-v^{\prime} a^{\prime}\left(y \widehat{\mathrm{id}}_{\beta}+u\right) Z_{1}-v^{\prime} \xi F_{1}, \\
& C_{2,1}=s z F_{1}, C_{2,2}=a^{\prime} s z F_{1}, C_{2,3}=-v\left(s z F_{1}\right)-v^{\prime} a^{\prime}\left(s z F_{1}\right),
\end{aligned}
$$

where $a^{\prime}=a+\mu^{\prime}, \mu=\mu^{\prime} s z$ and $\xi=\mu \sigma^{\prime}$. The challenge ciphertext $\widehat{\mathcal{C}}=$ $\left(C_{0}, C_{1,1}, C_{1,2}, C_{1,3}, C_{2,1}, C_{2,2}, C_{2,3}\right)$ is returned to $\mathscr{A}$. The computations below illustrate that $\widehat{\mathcal{C}}$ is a semi-functional encryption with $\sigma=\sigma^{\prime}+c d\left(y \widehat{\mathrm{id}}_{\beta}+u\right)$.

$$
\begin{aligned}
C_{1,2}=a^{\prime}\left(y \widehat{\mathrm{id}}_{\beta}+u\right) Z_{1}+\xi F_{1} & =\left(a+\mu^{\prime}\right)\left(y \widehat{\mathrm{id}}_{\beta}+u\right) c s d z F_{1}+\mu \sigma^{\prime} F_{1} \\
& =a\left(y \widehat{\mathrm{id}}_{\beta}+u\right) c s d z F_{1}+\mu^{\prime} h\left(\widehat{\mathbf{i d}}_{\beta}\right) c s d z F_{1}+\mu \sigma^{\prime} F_{1} \\
& =a s\left(\widehat{\operatorname{id}}_{\beta} Q_{1}+U_{1}\right)+\left(\mu^{\prime} s z\right)\left(c d\left(y \widehat{\mathrm{id}}_{\beta}+u\right)\right) F_{1}+\mu \sigma^{\prime} F_{1} \\
& =a s\left(\widehat{\operatorname{id}}_{\beta} Q_{1}+U_{1}\right)+\mu\left(c d\left(y \widehat{\mathrm{id}}_{\beta}+u\right)\right) F_{1}+\mu \sigma^{\prime} F_{1} \\
& =a s\left(\widehat{\operatorname{id}}_{\beta} Q_{1}+U_{1}\right)+\mu \sigma F_{1}
\end{aligned}
$$

Observe that $C_{1,1}=s\left(\widehat{\operatorname{id}}_{\beta} Q_{1}+U_{1}\right)=\left(c \cdot\left(y \widehat{\mathrm{id}}_{\beta}+u\right)\right)\left(s d z F_{1}\right)$. If $c=1$, then $\sigma=\sigma^{\prime}+d\left(y \widehat{\mathrm{id}}_{\beta}+u\right)$ and $\widehat{\mathcal{C}}$ is encrypted under $\widehat{\mathbf{i d}}_{\beta}$. Otherwise, $c$ is random, causing $\left(y \widehat{\mathrm{id}}_{\beta}+u\right)$ and consequently the target identity and $\sigma$ to be random quantities.

Guess: $\mathscr{A}$ returns its guess $\beta^{\prime}$ of $\beta$.

If the algorithm $\mathscr{B}$ returns 1 when $\beta=\beta^{\prime}$ and 0 otherwise, it can solve the A1 instance with advantage

$$
\operatorname{Adv}_{\mathcal{G}}^{\mathrm{A} 1}(\mathscr{B})=\mid \operatorname{Pr}\left[\beta=\beta^{\prime} \mid Z_{1} \text { is real }\right]-\operatorname{Pr}\left[\beta=\beta^{\prime} \mid Z_{1} \text { is random }\right]|=| \operatorname{Pr}\left[X_{M \text {-rand }}\right]-\operatorname{Pr}\left[X_{\text {final }}\right] \mid .
$$


Now, $\mathscr{A}$ 's advantage in winning the game is given by

$$
\begin{aligned}
\operatorname{Adv}_{\mathrm{LW}-\mathrm{IBE}}^{\text {ano-ind-cpa }}(\mathscr{A})=\left|\operatorname{Pr}\left[X_{\text {real }}\right]-\frac{1}{2}\right|= & \left|\operatorname{Pr}\left[X_{\text {real }}\right]-\operatorname{Pr}\left[X_{\text {final }}\right]\right| \\
\leq & \left|\operatorname{Pr}\left[X_{\text {real }}\right]-\operatorname{Pr}\left[X_{0}\right]\right|+\sum_{k=1}^{q}\left(\left|\operatorname{Pr}\left[X_{k-1}\right]-\operatorname{Pr}\left[X_{k}\right]\right|\right) \\
& +\left|\operatorname{Pr}\left[X_{q, 1}\right]-\operatorname{Pr}\left[X_{M-\text { rand }}\right]\right|+\left|\operatorname{Pr}\left[X_{M-\text { rand }}\right]-\operatorname{Pr}\left[X_{\text {final }}\right]\right| \\
\leq & \varepsilon_{\mathrm{LW} 1}+q \varepsilon_{\mathrm{LW} 2}+\varepsilon_{\mathrm{DBDH}-3}+\varepsilon_{\mathrm{A} 1}
\end{aligned}
$$

\section{Anonymous HIBE from LW-IBE}

In this section, we present our HIBE scheme, $\mathcal{L} \mathcal{W}$ - $\mathcal{A H I B \mathcal { E }}$, resulting from a BBG-type extension of the LW IBE scheme. A straightforward BBG-type extension would cause problems in simulating key extraction queries. We introduce some new elements to overcome this problem. The construction is based on a Type-3 prime-order pairing with group order $p$. Identities are variable length tuples of elements from $\mathbb{Z}_{p}^{\times}$with maximum length $h$.

The first step towards obtaining constant-size ciphertexts is to add elements $\left(Q_{1, j}\right)_{j \in[1, h]}, U_{1} \in$ $\mathbb{G}_{1}$ to the public parameters. These are used to create the identity hash - for an identity id = $\left(\mathrm{id}_{1}, \ldots, \mathrm{id}_{\ell}\right)$, the hash is given by $\sum_{j=1}^{\ell} \mathrm{id}_{j} Q_{1, j}+U_{1}$. This replaces the hash in LW-IBE without affecting the number of elements in the ciphertext. To facilitate key extraction, the corresponding elements in $\mathbb{G}_{2}$ also are provided. We introduce some notation here: the tuple $\left(P_{1},\left(Q_{1, j}\right)_{j \in[1, h]}, U_{1}\right)$ is denoted $\mathbf{Q}_{1}$ and let its $\mathbb{G}_{2}$ counterpart be $\mathbf{Q}_{2}$. Also present in the master secret of LW-IBE are the elements $V_{2}, V_{2}^{\prime}, F_{2}$ that provide cancellation analogous to the composite order setting. In the HIBE setting, these elements along with $\mathbf{Q}_{2}$, must be made public to assist in re-randomisation during delegation. Once these are made public, nothing is kept secret except for $\alpha$. This acts as a stumbling block against a dual system proof. In a proof within the dual system framework, some secret elements are used to create the so-called semi-functional components that are central to this proof methodology. In the composite order setting, this is achieved by keeping one subgroup hidden from the attacker which essentially forms the semi-functional space. In the prime order setting, making everything public leaks some information about the semi-functional components thus reducing the applicability of dual system method.

Our solution to this problem is to keep $\mathbf{Q}_{2}$ in the master secret. In a way, some elements of the group $\mathbb{G}_{2}$ are hidden and provide the basis for generating semi-functional components. To support delegation, suitably randomised copies of the key components are provided in the key itself. This technique was introduced by Boyen and Waters [4] to construct an anonymous HIBE scheme. $V_{2}, V_{2}^{\prime}, F_{2}$ are public to help in re-randomisation during delegation; this ensures proper distribution of the delegated key. Note that $\mathbf{Q}_{2}$ contains precisely the elements required to check whether a ciphertext is encrypted to a particular identity or not. A by-product of keeping this tuple secret is anonymity. Thus our scheme is secure in the ANO-IND-ID-CPA security model.

We now present the scheme $\mathcal{L} \mathcal{W}$ - $\mathcal{A H I B E}$. A discussion on the security of $\mathcal{L} \mathcal{W}-\mathcal{A H I B E}$ can be found in Section 5 .

\section{Construction:}

$\operatorname{Setup}(\kappa)$ : Let $h$ denote the maximum depth of the HIBE. Choose random generators $P_{1} \in \mathbb{G}_{1}$ and $P_{2} \in \mathbb{G}_{2}$; elements $Q_{1,1}, \ldots, Q_{1, h}, U_{1} \stackrel{\mathrm{U}}{\longleftarrow} \mathbb{G}_{1}$ and $Q_{2,1}, \ldots, Q_{2, h}, U_{2} \in \mathbb{G}_{2}$ such that $Q_{2, j} \sim Q_{1, j}$ for 
all $1 \leq j \leq h$ and $U_{2} \sim U_{1}$. Let $F_{2} \in \mathbb{G}_{2}$ be chosen at random and $v, v^{\prime}$ be chosen randomly from $\mathbb{Z}_{p}$. Set $V_{2}=v F_{2}, V_{2}^{\prime}=v^{\prime} F_{2}$. Pick $\alpha, a$ at random from $\mathbb{Z}_{p}$. Set $\tau=v+a v^{\prime}$ so that $\tau F_{2}=V_{2}+a V_{2}^{\prime}$.

$\mathcal{P P}:\left(P_{1}, a P_{1}, \tau P_{1}, U_{1}, a U_{1}, \tau U_{1},\left(Q_{1, j}, a Q_{1, j}, \tau Q_{1, j}\right)_{j \in[1, h]}, V_{2}, V_{2}^{\prime}, F_{2}, e\left(P_{1}, P_{2}\right)^{\alpha}\right)$.

$\mathcal{M S K}:\left(\alpha P_{2}, P_{2}, Q_{2,1}, \ldots, Q_{2, h}, U_{2}\right)$.

$\operatorname{Encrypt}\left(M, \mathbf{i d}=\left(\mathrm{id}_{1}, \ldots, \mathrm{id}_{\ell}\right), \mathcal{P} \mathcal{P}\right)$ : Choose $s \stackrel{\mathrm{U}}{\longleftarrow} \mathbb{Z}_{p}$. Let $\mathcal{H}_{i}(\mathbf{i d})=\mathrm{id}_{1} Q_{i, 1}+\cdots+\mathrm{id}_{\ell} Q_{i, \ell}+U_{i}$ for $i=1,2$. The ciphertext is given by $\mathcal{C}=\left(C_{0}, C_{1,1}, C_{1,2}, C_{1,3}, C_{2,1}, C_{2,2}, C_{2,3}\right)$ where the elements are computed as follows.

$$
\begin{aligned}
& C_{0}=M \times e\left(P_{1}, P_{2}\right)^{\alpha s}, \\
& C_{1,1}=s \mathcal{H}_{1}(\mathbf{i d}), C_{1,2}=a s \mathcal{H}_{1}(\mathbf{i d}), C_{1,3}=-\tau s \mathcal{H}_{1}(\mathbf{i d}) \\
& C_{2,1}=s P_{1}, C_{2,2}=a s P_{1}, C_{2,3}=-\tau s P_{1}
\end{aligned}
$$

$\operatorname{KeyGen}\left(\mathbf{i d}=\left(\mathrm{id}_{1}, \ldots, \mathrm{id}_{\ell}\right), \mathcal{M S} \mathcal{K}, \mathcal{P} \mathcal{P}\right)$ : Choose $w_{1}, w_{2}, r_{1}, r_{2}, r_{3}, r_{4},\left(z_{1, j}, z_{2, j}\right)_{j \in[\ell+1, h]} \stackrel{\mathrm{U}}{\longleftarrow} \mathbb{Z}_{p}$. The key consists of $6(n-\ell+2)$ group elements computed as follows.

$$
\begin{aligned}
& K_{1,1}=w_{1} P_{2}+r_{1} V_{2}, K_{1,2}=r_{1} V_{2}^{\prime}, K_{1,3}=r_{1} F_{2} \\
& K_{2,1}=\alpha P_{2}+w_{1} \mathcal{H}_{2}(\mathbf{i d})+r_{2} V_{2}, K_{2,2}=r_{2} V_{2}^{\prime}, K_{2,3}=r_{2} F_{2} \\
& D_{j, 1}=w_{1} Q_{2, j}+z_{1, j} V_{2}, D_{j, 2}=z_{1, j} V_{2}^{\prime}, D_{j, 3}=z_{1, j} F_{2} \text { for } \ell+1 \leq j \leq h \\
& J_{1,1}=w_{2} P_{2}+r_{3} V_{2}, J_{1,2}=r_{3} V_{2}^{\prime}, J_{1,3}=r_{3} F_{2} \\
& J_{2,1}=w_{2} \mathcal{H}_{2}(\mathbf{i d})+r_{4} V_{2}, J_{2,2}=r_{4} V_{2}^{\prime}, J_{2,3}=r_{4} F_{2} \\
& E_{j, 1}=w_{2} Q_{2, j}+z_{2, j} V_{2}, E_{j, 2}=z_{2, j} V_{2}^{\prime}, E_{j, 3}=z_{2, j} F_{2} \text { for } \ell+1 \leq j \leq h .
\end{aligned}
$$

The secret key for id is given by $\mathcal{S K}_{\mathbf{i d}}=\left(\mathcal{S}_{1}, \mathcal{S}_{2}\right)$, where $\mathcal{S}_{1}=\left(K_{1, i}, K_{2, i}, D_{j, i}\right)_{j \in[\ell+1, h], i=1,2,3}$ and $\mathcal{S}_{2}=\left(J_{1, i}, J_{2, i}, E_{j, i}\right)_{j \in[\ell+1, h], i=1,2,3}$. Notice that $\mathcal{S}_{2}$-components are almost same as $\mathcal{S}_{1}$-components except that the secret $\alpha$ is not embedded in $\mathcal{S}_{2}$. The set $\mathcal{S}_{2}$ is exclusively used for re-randomisation.

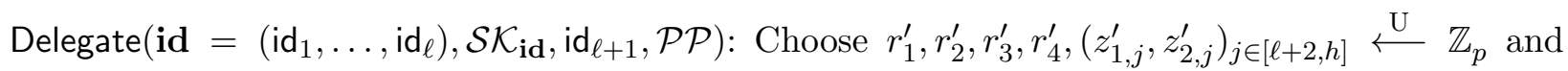
$w_{1}^{\prime}, w_{2}^{\prime} \stackrel{\mathrm{U}}{\longleftarrow} \mathbb{Z}_{p}^{\times}$. The components of the key for the identity id : id $\mathrm{i}_{\ell+1}$ are computed as follows.

$$
\begin{array}{lll}
K_{1,1} \leftarrow K_{1,1}+w_{1}^{\prime} J_{1,1}+r_{1}^{\prime} V_{2} & K_{2,1} \leftarrow K_{2,1}+\mathrm{id}_{\ell+1} D_{\ell+1,1}+w_{1}^{\prime}\left(J_{2,1}+\mathrm{id}_{\ell+1} E_{\ell+1,1}\right)+r_{2}^{\prime} V_{2} \\
K_{1,2} \leftarrow K_{1,2}+w_{1}^{\prime} J_{1,2}+r_{1}^{\prime} V_{2}^{\prime} & K_{2,2} \leftarrow K_{2,2}+\mathrm{id}_{\ell+1} D_{\ell+1,2}+w_{1}^{\prime}\left(J_{2,2}+\mathrm{id}_{\ell+1} E_{\ell+1,2}\right)+r_{2}^{\prime} V_{2}^{\prime} \\
K_{1,3} \leftarrow K_{1,3}+w_{1}^{\prime} J_{1,3}+r_{1}^{\prime} F_{2} & K_{2,3} \leftarrow K_{2,3}+\mathrm{id}_{\ell+1} D_{\ell+1,3}+w_{1}^{\prime}\left(J_{2,3}+\mathrm{id}_{\ell+1} E_{\ell+1,3}\right)+r_{2}^{\prime} F_{2} \\
& & \\
J_{1,1} \leftarrow w_{2}^{\prime} J_{1,1}+r_{3}^{\prime} V_{2} & J_{2,1} \leftarrow w_{2}^{\prime}\left(J_{2,1}+\mathrm{id}_{\ell+1} E_{\ell+1,1}\right)+r_{4}^{\prime} V_{2} \\
J_{1,2} \leftarrow w_{2}^{\prime} J_{1,2}+r_{3}^{\prime} V_{2}^{\prime} & J_{2,2} \leftarrow w_{2}^{\prime}\left(J_{2,2}+\mathrm{id}_{\ell+1} E_{\ell+1,2}\right)+r_{4}^{\prime} V_{2}^{\prime} \\
J_{1,3} \leftarrow w_{2}^{\prime} J_{1,3}+r_{3}^{\prime} F_{2} & J_{2,3} \leftarrow w_{2}^{\prime}\left(J_{2,3}+\mathrm{id}_{\ell+1} E_{\ell+1,3}\right)+r_{4}^{\prime} F_{2}
\end{array}
$$

For $j=\ell+2, \ldots, h$,

$$
\begin{array}{lll}
D_{j, 1} \leftarrow D_{j, 1}+w_{1}^{\prime} E_{j, 1}+z_{1, j}^{\prime} V_{2} & D_{j, 2} \leftarrow D_{j, 2}+w_{1}^{\prime} E_{j, 2}+z_{1, j}^{\prime} V_{2}^{\prime} & D_{j, 3} \leftarrow D_{j, 3}+w_{1}^{\prime} E_{j, 3}+z_{1, j}^{\prime} F_{2} \\
E_{j, 1} \leftarrow w_{2}^{\prime} E_{j, 1}+z_{2, j}^{\prime} V_{2} & E_{j, 2} \leftarrow w_{2}^{\prime} E_{j, 2}+z_{2, j}^{\prime} V_{2}^{\prime} & E_{j, 3} \leftarrow w_{2}^{\prime} E_{j, 3}+z_{2, j}^{\prime} F_{2}
\end{array}
$$

The above procedure essentially re-randomises all components of the key. As a result the distribution of a key obtained using delegation is the same as the distribution of a key obtained using the key generation procedure. To note the re-randomisation consider the following change of scalars for the modified key.

$$
w_{1} \leftarrow w_{1}+w_{1}^{\prime} w_{2} ; \quad w_{2} \leftarrow w_{2}^{\prime} w_{2} ;
$$




$$
\begin{array}{ll}
r_{1} \leftarrow r_{1}+r_{1}^{\prime}+w_{1}^{\prime} r_{3} ; & r_{3} \leftarrow w_{2}^{\prime} r_{3}+r_{3}^{\prime} ; \\
r_{2} \leftarrow r_{2}+r_{2}^{\prime}+\mathrm{id}_{\ell+1} z_{1, \ell+1}+w_{2}^{\prime}\left(r_{4}+\mathrm{id}_{\ell+1} z_{2, \ell+1}\right) ; & r_{4} \leftarrow w_{2}^{\prime}\left(r_{4}+\mathrm{id}_{\ell+1} z_{2, \ell+1}\right)+r_{4}^{\prime} ; \\
z_{1, j} \leftarrow z_{1, j}+z_{1, j}^{\prime}+w_{1}^{\prime} z_{2, j+1} \text { for } j=\ell+2, \ldots, h & z_{2, j} \leftarrow w_{2}^{\prime} z_{2, j}+z_{2, j}^{\prime} \text { for } j=\ell+2, \ldots, h
\end{array}
$$

These new randomisers are properly distributed by the choice of $w_{1}^{\prime}, w_{2}^{\prime}, r_{1}^{\prime}, r_{2}^{\prime}, r_{3}^{\prime}, r_{4}^{\prime},\left(z_{1, j}^{\prime}\right),\left(z_{2, j}^{\prime}\right)$. $\operatorname{Decrypt}\left(\mathcal{C}, \mathbf{i d}=\left(\mathrm{id}_{1}, \ldots, \mathrm{id}_{\ell}\right), \mathcal{S} \mathcal{K}_{\mathbf{i d}}, \mathcal{P} \mathcal{P}\right):$ Decryption is done as follows.

$$
M=C_{0} \times \frac{e\left(C_{1,1}, K_{1,1}\right) e\left(C_{1,2}, K_{1,2}\right) e\left(C_{1,3}, K_{1,3}\right)}{e\left(C_{2,1}, K_{2,1}\right) e\left(C_{2,2}, K_{2,2}\right) e\left(C_{2,3}, K_{2,3}\right)}
$$

Correctness of decryption of the HIBE scheme follows directly from that of LW-IBE since the decryption procedure remains the same - the additional delegation components do not play any role in decryption. Observe that computing the ratio of pairings in Equation (1) using $J_{1, i}, J_{2, i}$ $(i=1,2,3)$ instead of $K_{1, i}, K_{2, i}$ results in $1_{T}$ (the identity of $\left.\mathbb{G}_{T}\right)$.

\section{$5 \quad$ Security of $\mathcal{L W}-\mathcal{A H I B E}$}

We first provide some basic intuition underlying the proof with respect to different stages of security analysis (within the dual system framework), highlighting the similarities and differences with LWIBE security proof. Then, a detailed security analysis of $\mathcal{L W}-\mathcal{A H I B E}$ is presented in Section 5.2.

\subsection{Ideas Underlying the Security Proof}

The first step is to define semi-functional (sf) ciphertexts and keys. The definition of sf-ciphertext remains the same as LW-IBE. The keys of $\mathcal{L W}$ - $\mathcal{A H I B E}$ are significantly different from LW-IBE. We formulate the definition of sf-keys on the basis of the following observations.

- Sf-components for $\left(K_{1, i}, K_{2, i}\right)_{i=1,2}$ are identical to LW-IBE since only these components participate in decryption.

- It is required to define sf-components for $\left(D_{j, 1}, D_{j, 2}\right)_{j \in[\ell+1, h]}$ though they are only used during delegation to create the identity-hash. This is because they share the randomiser $w_{1}$ with $K_{1, i}, K_{2, i}$ and this randomiser comes from a problem instance in the reductions.

- Once sf-components are defined for $\mathcal{S}_{1}$, it is natural to ask: is it necessary to define sf-parts for $\mathcal{S}_{2}$ ? The answer is yes since otherwise the fourth reduction fails, where $P_{2}, U_{2},\left(Q_{2, j}\right)$ are masked by a quantity that forces the keys to be semi-functional. We have already seen this in the context of LW-IBE (see Theorem 1).

We would like to emphasise that the definition of semi-functional components (in both ciphertexts and keys), complexity assumptions and the reductions are all inter-linked. Changing the structure of sf-keys may determine the assumption required or affect simulation in some reduction. Also, for the reductions to go through, the sf-components may have to be defined in a particular way. The structure of sf-components we have is in a sense, optimal, subject to assumptions and simulations we provide.

An outline of the four main reductions in the augmented security proof (including anonymity) of LW-IBE is as follows.

First reduction: The goal of this reduction is to show that an attacker cannot distinguish between a normal ciphertext and an sf-ciphertext. It is achieved via a reduction from LW1 problem. An LW1 instance is embedded in the challenge ciphertext attempting to exploit the adversary's ability to detect the change in order to solve the problem. 
Second reduction: In this reduction, it is shown that if the adversary can decide whether the response to the $k$-th key extraction query is normal or semi-functional, then LW2 problem can be solved. The $k$-th key is constructed from an instance of LW2 problem in such a way that the key is normal if the instance is 'real' and semi-functional otherwise.

Third reduction: Here, the message that the challenge ciphertext encrypts, is changed to random element of $\mathbb{G}_{T}$. It is shown that solving the DBDH-3 problem is no harder than distinguishing between an sf-encryption of the real message from an sf-encryption of a random element of $\mathbb{G}_{T}$.

Fourth reduction: Challenge ciphertext encrypts a random message under a random identity. The identity-hash is created using the challenge in an instance of A1 problem thus making it real or random according to the distribution of the challenge.

This strategy does not directly extend to the hierarchical setting. Several challenges/restrictions emerge as we try to prove security of $\mathcal{L W}$ - $\mathcal{A H I B \mathcal { E }}$.

The first and the third reductions for $\mathcal{L W}-\mathcal{A H I B E}$ are the closest to the corresponding reductions for LW-IBE appearing in [12]. In these reductions, the simulations of the public parameters; the ciphertext elements; and the components of the key which are present in LW-IBE; are exactly the same as for LW-IBE. The only technicality is to ensure that the extra components of the key can be properly simulated without changing the corresponding assumptions (LW1 for the first reduction and DBDH-3 for the third reduction).

The second reduction presents some technical novelty. We need to extend the dual-system technique to handle this reduction. In this reduction, it is shown that the adversary cannot decide whether the response to the $k$-th key extraction query is normal or semi-functional. Compared to the LW-IBE, the key has additional components which are required for delegation and rerandomisation; moreover, these have semi-functional parts. A new technique is required to handle these simulations.

Partial semi-functionality: Consider the second reduction where the $k$-th key is made semifunctional. LW-IBE reduction embeds a pairwise independent function in the $k$-th key as well as the challenge ciphertext to ensure proper distribution of the scalars involved in the sf-components. This function is determined by the elements used to create the identity-hash. An attempt to use the same strategy for $\mathcal{L W}-\mathcal{A H I B E}$, however, causes a problem. The reason is that both $\mathcal{S}_{1}$ and $\mathcal{S}_{2}$ of the $k$-th key have sf-components and the identity-hash is now present in three places - challenge ciphertext, $\mathcal{S}_{1}$ and $\mathcal{S}_{2}$. One possible way to get around this problem is to embed a 3 -wise independent function but this will blow up the size of the public parameters. The other way to get around the problem is to use two separate instances to generate the two parts of the key so that they have the proper distribution when semi-functional. In order to do this, we need to change the key from normal to semi-functional in two steps - first make $\mathcal{S}_{1}$ semi-functional followed by $\mathcal{S}_{2}$. We call a key partial semi-functional if $\mathcal{S}_{1}$ is semi-functional and $\mathcal{S}_{2}$ is normal.

The second step of the dual-system technique changes the key in the $k$-th response from normal to semi-functional (without the adversary noticing this). In our case, this is done in two sub-steps - the first step changes from normal to partial semi-functional and the second step changes from partial semi-functional to semi-functional. This leads to a slight degradation in the security bound by a factor of 2 .

The fourth reduction is to show anonymity of the HIBE scheme. This is almost the same as the reduction that we have provided to show the anonymity of the LW-IBE. The only difference is that the extra elements of the key have to properly simulated. 


\subsection{Detailed Proof}

As is typical in the dual-system technique, we first describe semi-functional ciphertexts and keys. These are required only in the reductions and not in the actual scheme.

Semi-functional ciphertext: Let $C_{0}^{\prime}, C_{1,1}^{\prime}, C_{1,2}^{\prime}, C_{1,3}^{\prime}, C_{2,1}^{\prime}, C_{2,2}^{\prime}, C_{2,3}^{\prime}$ be ciphertext elements normally generated by the Encrypt algorithm for message $M$ and identity id. Let $V_{1}^{\prime}, F_{1}$ be elements of $\mathbb{G}_{1}$ such that $V_{1}^{\prime} \sim V_{2}^{\prime}$ and $F_{1} \sim F_{2}$. Choose $\mu, \sigma \in \mathbb{Z}_{p}$ at random. The semi-functional ciphertext generation algorithm will modify the normal ciphertext as: $C_{0}=C_{0}^{\prime}, C_{1,1}=C_{1,1}^{\prime}, C_{2,1}=C_{2,1}^{\prime}$ and

$$
C_{1,2}=C_{1,2}^{\prime}+\mu \sigma F_{1}, C_{1,3}=C_{1,3}^{\prime}-\mu \sigma V_{1}^{\prime}, C_{2,2}=C_{2,2}^{\prime}+\mu F_{1}, C_{2,3}=C_{2,3}^{\prime}-\mu V_{1}^{\prime} .
$$

Semi-functional key: Let $\left(\mathcal{S}_{1}, \mathcal{S}_{2}\right)$ be the secret key generated by the KeyGen algorithm for identity id $=\left(\mathrm{id}_{1}, \ldots\right.$, id $\left._{\ell}\right)$ with $\mathcal{S}_{1}=\left(K_{1, i}, K_{2, i}, D_{j, i}\right)_{j \in[\ell+1, h], i=1,2,3}, \mathcal{S}_{2}=\left(J_{1, i}, J_{2, i}, E_{j, i}\right)_{j \in[\ell+1, h], i=1,2,3}$. Let $\gamma_{1}, \pi, \gamma_{2}, \eta,\left(\pi_{j}, \eta_{j}\right)_{j \in[\ell+1, h]}$ be uniform random elements chosen from $\mathbb{Z}_{p}$. The semi-functional key generation algorithm will modify the normal key as:

$$
\begin{array}{ll}
K_{1,1}=K_{1,1}-a \gamma_{1} F_{2}, K_{1,2}=K_{1,2}+\gamma_{1} F_{2}, & J_{1,1}=J_{1,1}-a \gamma_{2} F_{2}, J_{1,2}=J_{1,2}+\gamma_{2} F_{2}, \\
K_{2,1}=K_{2,1}-a \gamma_{1} \pi F_{2}, K_{2,2}=K_{2,2}+\gamma_{1} \pi F_{2}, & J_{2,1}=J_{2,1}-a \gamma_{2} \eta F_{2}, J_{2,2}=J_{2,2}+\gamma_{2} \eta F_{2}, \\
\text { For } j=\ell+1, \ldots, h & \\
D_{j, 1}=D_{j, 1}-a \gamma_{1} \pi_{j} F_{2}, D_{j, 2}=D_{j, 2}+\gamma_{1} \pi_{j} F_{2}, & E_{j, 1}=E_{j, 1}-a \gamma_{2} \eta_{j} F_{2}, E_{j, 2}=E_{j, 2}+\gamma_{2} \eta_{j} F_{2} .
\end{array}
$$

The rest of the components remain unchanged.

Partial semi-functional key: In a partial semi-functional key, $\mathcal{S}_{2}$ is normal and $\mathcal{S}_{1}$ is semi-functional.

Note that definitions are similar to [12] except for the delegation and re-randomisation components. Since decryption is not affected by these components of the key, all the requirements for semi-functional keys and ciphertexts are satisfied. A pair of semi-functional ciphertext and key is called nominally semi-functional if $\sigma=\pi$ (condition that makes decryption successful).

Structure of the Proof. We consider the security model defined in Section 2.5. The proof is organised as a hybrid over a sequence of $2 q+4$ games defined as follows.

Game $_{\text {real }}$ : ano-ind-cpa game defined in Section 2.5.

Game $_{0}$ : the challenge ciphertext is semi-functional and all the keys returned to the adversary are normal.

Game $_{k, 0}$ (for $1 \leq k \leq q$ ): $k$-th key is partial semi-functional, the first $k-1$ keys are semi-functional; the rest of the keys are normal.

Game $_{k, 1}$ (for $1 \leq k \leq q$ ): similar to Game $k, 0$ except that the $k$-th key is (fully) semi-functional.

Game $_{M-\text { rand }}$ all keys are semi-functional and the challenge ciphertext encrypts a random message to the challenge identity.

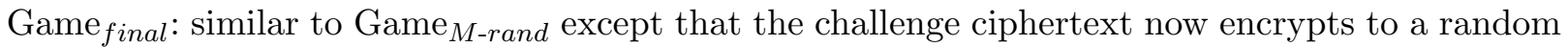
identity vector.

These games are ordered as Game real $_{\text {, Game }}$, Game $_{1,0}$, Game $_{1,1}, \ldots$, Game $_{q, 0}$, Game $_{q, 1}$, Game $_{M \text {-rand }}$, Game $_{\text {final }}$ in our hybrid argument. Let $X_{\text {real }}, X_{0}, X_{k, 0}, X_{k, 1}($ for $k=0,1, \ldots, q$ ), $X_{M \text {-rand }}$ and $X_{\text {final }}$ be events that $\mathscr{A}$ wins in $\mathrm{Game}_{r e a l}, \mathrm{Game}_{0}, \mathrm{Game}_{k, 0}, \mathrm{Game}_{k, 1}$, Game $_{M \text {-rand }}$

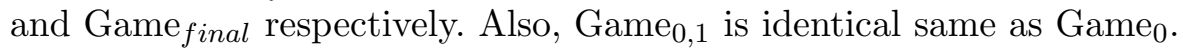


For the proof it will be convenient to use the following short-hand: denote by $h(\mathbf{i d})$ the sum $\sum_{j=1}^{\ell} y_{j} \mathrm{id}_{j}+u$ and by $g(\mathbf{i d})$ the sum $\sum_{j=1}^{\ell} \lambda_{j} \mathrm{id}_{j}+\nu$, where $y_{1}, \ldots, y_{n}, u, \lambda_{1}, \ldots, \lambda_{n}, \nu$ are elements of $\mathbb{Z}_{p}$ to be chosen in the proofs.

Theorem 2. If the $\left(\varepsilon_{\mathrm{LW} 1}, t^{\prime}\right)$-LW1, $\left(\varepsilon_{\mathrm{LW} 2}, t^{\prime}\right)$-LW2, $\left(\varepsilon_{\mathrm{DBDH}-3}, t^{\prime}\right)$-DBDH-3 and $\left(\varepsilon_{\mathrm{A} 1}, t^{\prime}\right)-\mathrm{A} 1$ assumptions hold, then $\mathcal{L W}$-AHIBE is $(\varepsilon, t, q)$-ANO-IND-ID-CPA secure where

$$
\varepsilon \leq \varepsilon_{\mathrm{LW} 1}+2 q \varepsilon_{\mathrm{LW} 2}+\varepsilon_{\mathrm{DBDH}-3}+\varepsilon_{\mathrm{A} 1}
$$

and $t=t^{\prime}-O(q \rho)$, where $\rho$ is an upper bound on the time required for one scalar multiplication in $\mathbb{G}_{1}$ or $\mathbb{G}_{2}$.

Proof. For any $t$-time adversary $\mathscr{A}$ against $\mathcal{L W}$ - $\mathscr{A H I B E}$ in the ano-ind-cpa, its advantage in winning the game is given by

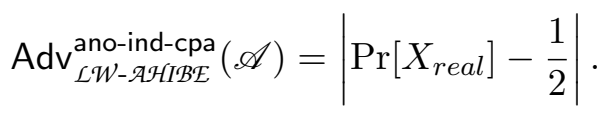

We know that $\operatorname{Pr}\left[X_{\text {final }}\right]=\frac{1}{2}$ and hence we have

$$
\begin{aligned}
& \operatorname{Adv}_{\mathcal{L W} \text { - } \mathscr{H} \mathcal{H}[\mathcal{B E E}}^{\text {ano-ind }}(\mathscr{A})=\left|\operatorname{Pr}\left[X_{\text {real }}\right]-\operatorname{Pr}\left[X_{\text {final }}\right]\right| \\
& \leq\left|\operatorname{Pr}\left[X_{\text {real }}\right]-\operatorname{Pr}\left[X_{0}\right]\right|+\sum_{k=1}^{q}\left(\left|\operatorname{Pr}\left[X_{k-1,1}\right]-\operatorname{Pr}\left[X_{k, 0}\right]\right|\right)+\sum_{k=1}^{q}\left(\left|\operatorname{Pr}\left[X_{k, 0}\right]-\operatorname{Pr}\left[X_{k, 1}\right]\right|\right) \\
& +\left|\operatorname{Pr}\left[X_{q, 1}\right]-\operatorname{Pr}\left[X_{M-\text { rand }}\right]\right|+\left|\operatorname{Pr}\left[X_{M-\text { rand }}\right]-\operatorname{Pr}\left[X_{\text {final }}\right]\right| \\
& \leq \varepsilon_{\mathrm{LW} 1}+2 q \varepsilon_{\mathrm{LW} 2}+\varepsilon_{\mathrm{DBDH}-3}+\varepsilon_{\mathrm{A} 1}
\end{aligned}
$$

The last inequality follows from the lemmas $1,2,3,4$ and 5 . In all the lemmas, $\mathscr{A}$ is a $t$-time adversary against $\mathcal{L W}$ - $\mathcal{A H I B \mathcal { E }}$ and $\mathscr{B}$ is an algorithm running in time $t^{\prime}$ that interacts with $\mathscr{A}$ and solves one of the three problems LW1, LW2, DBDH-3 or A1.

Lemma 1. $\left|\operatorname{Pr}\left[X_{\text {real }}\right]-\operatorname{Pr}\left[X_{0}\right]\right| \leq \varepsilon_{\mathrm{LW} 1}$.

Proof. The algorithm $\mathscr{B}$ receives the following instance of LW1

$$
\left(F_{1}, b s F_{1}, s F_{1}, a F_{1}, a b^{2} F_{1}, b F_{1}, b^{2} F_{1}, a s F_{1}, b^{2} s F_{1}, b^{3} F_{1}, b^{3} s F_{1}, F_{2}, b F_{2}, Z_{1}\right) .
$$

$\mathscr{B}$ has to determine whether $Z_{1}=a b^{2} s F_{1}$ or $Z_{1} \in \in_{\mathrm{U}} \mathbb{G}_{1}$. We will call $Z_{1}$ "real" in the former case and "random" otherwise. $\mathscr{B}$ simulates the security game as described below.

Set-Up: $\mathscr{B}$ chooses $\alpha, y, v^{\prime},\left(y_{j}, \lambda_{j}\right)_{j \in[1, h]}, u, \nu \stackrel{\mathrm{U}}{\longleftarrow} \mathbb{Z}_{p}$ and sets the parameters.

$$
\begin{gathered}
P_{1}=b^{2} F_{1}+y F_{1}, Q_{1, j}=\lambda_{j}\left(b^{2} F_{1}\right)+y_{j} F_{1} \text { for } 1 \leq j \leq h, U_{1}=\nu\left(b^{2} F_{1}\right)+u F_{1} \\
V_{2}=b F_{2}, V_{2}^{\prime}=v^{\prime} F_{2} .
\end{gathered}
$$

This implicitly sets $P_{2}=\left(b^{2}+y\right) F_{2}, v=b$ and $\tau=b+a v^{\prime}$. Compute $a P_{1}=a b^{2} F_{1}+y\left(a F_{1}\right)$ and $\tau P_{1}=b^{3} F_{1}+v^{\prime}\left(a b^{2} F_{1}\right)+y\left(b F_{1}\right)+y v^{\prime}\left(a F_{1}\right)$. The elements $\left(a Q_{1, j}, \tau Q_{1, j}\right)_{j \in[1, h]}, a U_{1}, \tau U_{1}$ are constructed similarly. Set $e\left(P_{1}, P_{2}\right)^{\alpha}=\left(e\left(b^{3} F_{1}+y\left(b F_{1}\right), b F_{2}\right) e\left(P_{1}, y F_{2}\right)\right)^{\alpha}$. The simulator gives the following public parameters to $\mathscr{A}$.

$$
\mathcal{P P}=\left(P_{1}, Q_{1,1}, \ldots, Q_{1, h}, U_{1}, a P_{1}, a Q_{1,1}, \ldots, a Q_{1, h}, a U_{1}, \tau P_{1}, \tau Q_{1,1}, \ldots, \tau Q_{1, h}, \tau U_{1}, e\left(P_{1}, P_{2}\right)^{\alpha}\right) .
$$

Phases 1 and 2: $\mathscr{A}$ makes a number of key extract queries. $\mathscr{B}$ does not know $P_{2}, Q_{2, j}, U_{2}$ which are part of the master secret. The secret key for a query on id is constructed as follows. $\mathscr{B}$ chooses $r_{1}^{\prime}, r_{2}^{\prime}, r_{3}^{\prime}, r_{4}^{\prime},\left(z_{1, j}^{\prime}, z_{2, j}^{\prime}\right)_{j \in[\ell+1, h]}, w_{1}, w_{2} \in \mathbb{Z}_{p}$ at random and computes 
$K_{1,1}=w_{1} y F_{2}+r_{1}^{\prime}\left(b F_{2}\right), K_{1,3}=r_{1}^{\prime} F_{2}-w\left(b F_{2}\right), K_{1,2}=v^{\prime} K_{1,3}$,

$K_{2,1}=\alpha y F_{2}+r_{2}^{\prime}\left(b F_{2}\right)+w h(\mathbf{i d}) F_{2}, K_{2,3}=r_{2}^{\prime} F_{2}-\left(w_{1} g(\mathbf{i d})+\alpha\right)\left(b F_{2}\right), K_{2,2}=v^{\prime} K_{2,3},$,

$D_{j, 1}=w_{1} y_{j} F_{2}+z_{1, j}^{\prime}\left(b F_{2}\right), D_{j, 3}=z_{1, j}^{\prime} F_{2}-w_{1} \lambda_{j}\left(b F_{2}\right), D_{j, 2}=v^{\prime} D_{j, 3}$ for $\ell+1 \leq j \leq h$,

$J_{1,1}=w_{2} y F_{2}+r_{3}^{\prime}\left(b F_{2}\right), J_{1,3}=r_{3}^{\prime} F_{2}-w_{2}\left(b F_{2}\right), J_{1,2}=v^{\prime} J_{1,3}$,

$J_{2,1}=r_{4}^{\prime}\left(b F_{2}\right)+w_{2} h(\mathbf{i d}) F_{2}, J_{2,3}=r_{4}^{\prime} F_{2}-w_{2} g(\mathbf{i d})\left(b F_{2}\right), J_{2,2}=v^{\prime} J_{2,3}$,

$E_{j, 1}=w_{2} y_{j} F_{2}+z_{2, j}^{\prime}\left(b F_{2}\right), E_{j, 3}=z_{2, j}^{\prime} F_{2}-w_{2} \lambda_{j}\left(b F_{2}\right), E_{j, 2}=v^{\prime} E_{j, 3}$ for $\ell+1 \leq j \leq h$,

implicitly setting

$$
\begin{aligned}
& r_{1}=r_{1}^{\prime}-w_{1} b, r_{2}=r_{2}^{\prime}-\left(w_{1} g(\mathbf{i d})+\alpha\right) b, \\
& r_{3}=r_{3}^{\prime}-w_{2} b, r_{4}=r_{4}^{\prime}-w_{2} g(\mathbf{i d}) b, \\
& z_{1, j}=z_{1, j}^{\prime}-w_{1} \lambda_{j} b, z_{2, j}=z_{2, j}^{\prime}-w_{2} \lambda_{j} b \text { for } j=\ell+1, \ldots, h .
\end{aligned}
$$

The following computation shows that the components are well-formed.

$$
\begin{aligned}
K_{1,1} & =w y F_{2}+r_{1}^{\prime}\left(b F_{2}\right) \\
& =w y F_{2}+\left(r_{1}+w b\right) b F_{2} \\
& =w\left(y F_{2}+b^{2} F_{2}\right)+r_{1}\left(b F_{2}\right) \\
& =w P_{2}+r_{1} V_{2} \\
D_{1, j} & =w y_{j} F_{2}+z_{j}^{\prime}\left(b F_{2}\right) \\
& =w y_{j} F_{2}+\left(z_{1, j}+w \lambda_{j} b\right)\left(b F_{2}\right) \\
& =w\left(y_{j} F_{2}+\lambda_{j} b^{2} F_{2}\right)+z_{1, j}\left(b F_{2}\right) \\
& =w Q_{2, j}+z_{1, j} V_{2}
\end{aligned}
$$$$
K_{2,1}=\alpha y F_{2}+r_{2}^{\prime}\left(b F_{2}\right)+w h(\mathbf{i d}) F_{2}
$$$$
=\alpha y F_{2}+\left(r_{2}+(w g(\mathbf{i d})+\alpha) b\right) b F_{2}+w h(\mathbf{i d}) F_{2}
$$$$
=\alpha\left(y F_{2}+b^{2} F_{2}\right)+r_{2}\left(b F_{2}\right)+w\left(g(\mathbf{i d}) b^{2} F_{2}+h(\mathbf{i d}) F_{2}\right)
$$$$
=\alpha P_{2}+w \mathcal{H}_{2}(\mathbf{i d})+r_{2} V_{2}
$$

Following the same logic, it can be verified that $J_{1,1}, J_{2,1}, E_{j, 1}$ are well-formed. Remaining components clearly have the right form.

Challenge: $\mathscr{B}$ receives two pairs $\left(M_{0}, \widehat{\mathbf{i d}}_{0}\right)$ and $\left(M_{1}, \widehat{\mathbf{i d}}_{1}\right)$ from $\mathscr{A}$. It chooses $\beta \in\{0,1\}$ at random. $\mathscr{B}$ computes the ciphertext for $M_{\beta}$ under $\widehat{\mathbf{i d}}_{\beta}$ as follows.

$$
\begin{aligned}
& C_{0}=M_{\beta} \cdot\left(e\left(b^{3} s F_{1}+y\left(b s F_{1}\right), b F_{2}\right) e\left(b^{2} s F_{1}+y\left(s F_{1}\right), y F_{2}\right)\right)^{\alpha}=M_{\beta} \cdot e\left(P_{1}, P_{2}\right)^{\alpha s} \\
& C_{1,1}=g\left(\widehat{\mathbf{i d}}_{\beta}\right)\left(b^{2} s F_{1}\right)+h\left(\widehat{\mathbf{i d}}_{\beta}\right)\left(s F_{1}\right), C_{1,2}=g\left(\widehat{\mathbf{i d}}_{\beta}\right) Z_{1}+h\left(\widehat{\mathbf{i d}}_{\beta}\right)\left(a s F_{1}\right) \\
& C_{1,3}=-g\left(\hat{\mathbf{i d}}_{\beta}\right)\left(b^{3} s F_{1}\right)-h\left(\widehat{\mathbf{i d}}_{\beta}\right)\left(b s F_{1}\right)-v^{\prime} g\left(\widehat{\mathbf{i d}}_{\beta}\right) Z_{1}-v^{\prime} h\left(\hat{\mathbf{i d}}_{\beta}\right)\left(a s F_{1}\right) \\
& C_{2,1}=b^{2} s F_{1}+y\left(s F_{1}\right), C_{2,2}=Z_{1}+y\left(a s F_{1}\right) \\
& C_{2,3}=-b^{3} s F_{1}-y\left(b s F_{1}\right)-v^{\prime} Z_{1}-v^{\prime}\left(a s F_{1}\right) .
\end{aligned}
$$

$\mathscr{B}$ returns $\widehat{\mathcal{C}}=\left(C_{0}, C_{1,1}, C_{1,2}, C_{1,3}, C_{2,1}, C_{2,2}, C_{2,3}\right)$ to $\mathscr{A}$.

If $Z_{1}=a b^{2} s F_{1}$, then it is easy to see that $\widehat{\mathcal{C}}$ is normal. Otherwise, $Z_{1}=\left(a b^{2} s+\mu\right) F_{1}$ for some $\mu \in \in_{\mathrm{U}} \mathbb{Z}_{p}$ and $\widehat{\mathcal{C}}$ is semi-functional with $\mu=\mu$ and $\sigma=g\left(\widehat{\mathbf{i d}}_{\beta}\right)$. The calculation below shows that $C_{1,2}$ is a properly formed (semi-functional) component.

$$
\begin{aligned}
C_{1,2} & =g\left(\widehat{\mathbf{i d}}_{\beta}\right) Z_{1}+h\left(\widehat{\mathbf{i d}}_{\beta}\right)\left(a s F_{1}\right) \\
& =g\left(\widehat{\mathbf{i d}}_{\beta}\right)\left(a b^{2} s+\mu\right) F_{1}+h\left(\widehat{\mathbf{i d}}_{\beta}\right)\left(a s F_{1}\right) \\
& =a s\left(g\left(\widehat{\mathbf{i d}}_{\beta}\right) b^{2} F_{1}+h\left(\widehat{\mathbf{i d}}_{\beta}\right) F_{1}\right)+\mu g\left(\widehat{\mathbf{i d}}_{\beta}\right) F_{1} \\
& =a s \mathcal{H}_{1}\left(\widehat{\mathbf{i d}}_{\beta}\right)+\mu \sigma F_{1}
\end{aligned}
$$


Verification of the well-formedness of $C_{1,3}, C_{2,2}$ and $C_{2,3}$ follows the same pattern. Scalars $\left(\lambda_{j}\right)_{j \in[1, h]}, \nu$ are information theoretically hidden from $\mathscr{A}$ 's view and hence $\sigma=g($ id $)$ appears to be uniformly and independently distributed with respect to all other information provided to $\mathscr{A}$.

Note that, to check whether $\widehat{\mathcal{C}}$ is semi-functional or not, $\mathscr{B}$ itself could try to decrypt it with a semi-functional key for $\widehat{\mathbf{i d}}_{\beta}$. Any such attempt will fail due to the following reason $-a F_{2}$ is unavailable to $\mathscr{B}$; it could try to cancel out $-a \gamma F_{2}$ in $K_{1,1}$ or $\gamma F_{2}$ in $K_{1,2}$ with some other elements; but we do not see how to achieve this keeping the link between $K_{1,1}$ and $K_{1,2}$ (via $\gamma$ ) intact, without knowing $a F_{2}$.

Guess: The adversary returns its guess $\beta^{\prime}$ to $\mathscr{B}$.

If $Z_{1}$ is real, $\widehat{\mathcal{C}}$ is normal and hence $\mathscr{B}$ simulates Game real. Otherwise, $Z_{1}$ is random and $\widehat{\mathcal{C}}$

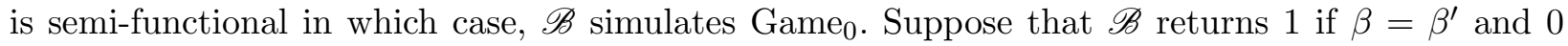
otherwise. Then it can solve the LW1 problem with advantage

$$
\operatorname{Adv}_{\mathcal{G}}^{\mathrm{LW} 1}(\mathscr{B})=\mid \operatorname{Pr}\left[\beta=\beta^{\prime} \mid Z_{1} \text { is real }\right]-\operatorname{Pr}\left[\beta=\beta^{\prime} \mid Z_{1} \text { is random }\right]|=| \operatorname{Pr}\left[X_{\text {real }}\right]-\operatorname{Pr}\left[X_{0}\right] \mid \leq \varepsilon_{\mathrm{LW1} 1} .
$$

Lemma 2. $\left|\operatorname{Pr}\left[X_{k-1,1}\right]-\operatorname{Pr}\left[X_{k, 0}\right]\right| \leq \varepsilon_{\mathrm{LW} 2}$ for $1 \leq k \leq q$.

Proof. Let $\left(F_{1}, d F_{1}, d^{2} F_{1}, b x F_{1}, d b x F_{1}, d^{2} x F_{1}, F_{2}, d F_{2}, b F_{2}, c F_{2}, Z_{2}\right)$ be the instance of LW2 that $\mathscr{B}$ receives. Let $Z_{2}=(b c+\gamma) F_{2}$. $\mathscr{B}$ 's task is to decide whether $\gamma=0\left(Z_{2}\right.$ is real $)$ or $\gamma \in \in_{\mathrm{U}} \mathbb{Z}_{p}\left(Z_{2}\right.$ is random).

Set-Up: $\mathscr{B}$ chooses $\alpha, a, y_{v}, y_{1}, \ldots, y_{h}, u, \lambda_{1}, \ldots, \lambda_{h}, \nu \stackrel{\mathrm{U}}{\longleftarrow} \mathbb{Z}_{p}$ and computes parameters as follows. $P_{1}=d F_{1}, Q_{1, j}=\lambda_{j}\left(d F_{1}\right)+y_{j} F_{1}$ for $1 \leq j \leq h, U_{1}=\nu\left(d F_{1}\right)+u F_{1}, V_{2}=-a\left(b F_{2}\right)+d F_{2}+y_{v} F_{2}$ and $V_{2}^{\prime}=b F_{2}$ setting $v=-a b+d+y_{v}, v^{\prime}=b$ and $\tau=d+y_{v}$. The element $\tau P_{1}$ can be computed as $\tau P_{1}=d^{2} F_{1}+y_{v}\left(d F_{1}\right)$. The parameters $\tau Q_{1, j}$ for $1 \leq j \leq h$ and $\tau U_{1}$ are given by $\tau Q_{1, j}=$ $\lambda_{j}\left(d^{2} F_{1}\right)+y_{j}\left(d F_{1}\right)+y_{v} \lambda_{j}\left(d F_{1}\right)+y_{v} y_{j} F_{1}$ and $\tau U_{1}=\nu\left(d^{2} F_{1}\right)+u\left(d F_{1}\right)+y_{v} \nu\left(d F_{1}\right)+y_{v} u F_{1}$. The remaining parameters required to provide $\mathcal{P} \mathcal{P}$ to $\mathscr{A}$ are computed using $a, \alpha$ and elements of the problem instance. Elements of the master secret key can also be obtained from the instance and randomisers chosen at setup.

Phases 1 and 2: The key extraction queries for identities $\mathbf{i d}_{1}, \ldots, \mathbf{i d}_{q}$ are answered in the following way. If $i<k$, a semi-functional key is returned and if $i>k$ a normal key is returned. $\mathscr{B}$ creates semi-functional keys using the master secret, $a$ and $F_{2}$.

For $i=k, \mathscr{B}$ computes of $\mathcal{S}_{1}$ using the problem instance in the following manner. Let $\mathbf{i d}_{k}=$ $\left(\mathrm{id}_{1}, \ldots, \mathrm{id}_{\ell}\right) . \mathscr{B}$ chooses $w_{1}^{\prime}, r_{2}^{\prime}, z_{1, \ell+1}^{\prime}, \ldots, z_{1, h}^{\prime} \stackrel{\mathrm{U}}{\longleftarrow} \mathbb{Z}_{p}$.

$$
\begin{aligned}
& K_{1,1}=w_{1}^{\prime} P_{2}-a Z_{2}+y_{v}\left(c F_{2}\right), K_{1,2}=Z_{2}, K_{1,3}=c F_{2} \\
& K_{2,1}=\alpha P_{2}+w_{1}^{\prime}\left(g\left(\mathbf{i d}_{k}\right)\left(d F_{2}\right)+h\left(\mathbf{i d}_{k}\right) F_{2}\right)+r_{2}^{\prime} V_{2}-a g\left(\mathbf{i d}_{k}\right) Z_{2}+y_{v} g\left(\mathbf{i d}_{k}\right)\left(c F_{2}\right)-h\left(\mathbf{i d}_{k}\right) c F_{2} \\
& K_{2,2}=r_{2}^{\prime} V_{2}^{\prime}+g\left(\mathbf{i d}_{k}\right) Z_{2}, K_{2,3}=r_{2}^{\prime} F_{2}+g\left(\mathbf{i d}_{k}\right)\left(c F_{2}\right)
\end{aligned}
$$

and for $j=\ell+1, \ldots, h$, set

$$
\begin{aligned}
& D_{j, 1}=w_{1}^{\prime} Q_{2, j}+z_{1, j}^{\prime} V_{2}-y_{j}\left(c F_{2}\right)-a \lambda_{j} Z_{2}+y_{v} \lambda_{j}\left(c F_{2}\right) \\
& D_{j, 2}=z_{1, j}^{\prime} V_{2}^{\prime}+\lambda_{j} Z_{2}, D_{j, 3}=z_{1, j}^{\prime} F_{2}+\lambda_{j}\left(c F_{2}\right)
\end{aligned}
$$

thus implicitly setting $w_{1}=w_{1}^{\prime}-c, r_{1}=c, r_{2}=r_{2}^{\prime}+g\left(\mathbf{i d}_{k}\right) c$ and $z_{1, j}=z_{1, j}^{\prime}+\lambda_{j} c$ for $\ell+1 \leq j \leq h$. 
Let $\mathcal{S}_{1}=\left(K_{1, i}, K_{2, i}, D_{j, i}\right)_{j \in[\ell+1, h], i=1,2,3}$. The second set $\mathcal{S}_{2}=\left(J_{1, i}, J_{2, i}, E_{j, i}\right)_{j \in[\ell+1, h], i=1,2,3}$ is created normally. $\mathscr{B}$ returns $\mathcal{S} \mathcal{K}_{\mathbf{i d}_{k}}=\left(\mathcal{S}_{1}, \mathcal{S}_{2}\right)$ as the key for $\mathbf{i d}_{k}$. If $Z_{2}=b c F_{2}$ then the key for $\mathbf{i d}_{k}$ is normal. We show that $K_{2,1}$ is well-formed. Verifying the remaining parts can be done analogously.

$$
\begin{aligned}
K_{2,1}= & \alpha P_{2}+w_{1}^{\prime}\left(g\left(\mathbf{i d}_{k}\right)\left(d F_{2}\right)+h\left(\mathbf{i d}_{k}\right) F_{2}\right)+r_{2}^{\prime} V_{2}-a g\left(\mathbf{i d}_{k}\right) Z_{2}+y_{v} g\left(\mathbf{i d}_{k}\right)\left(c F_{2}\right)-h\left(\mathbf{i d}_{k}\right) c F_{2} \\
= & \alpha P_{2}+\left(w_{1}+c\right) \mathcal{H}_{2}\left(\mathbf{i d}_{k}\right)+\left(r_{2}-g\left(\mathbf{i d}_{k}\right) c\right)\left(-a\left(b F_{2}\right)+d F_{2}+y_{v} F_{2}\right) \\
& \quad+g\left(\mathbf{i d}_{k}\right)\left(-a b c F_{2}+y_{v} c F_{2}\right)-h\left(\mathbf{i d}_{k}\right) c F_{2} \\
= & \alpha P_{2}+\left(w_{1}+c\right) \mathcal{H}_{2}\left(\mathbf{i d}_{k}\right)+r_{2} V_{2}-g\left(\mathbf{i d}_{k}\right) c d F_{2}+g\left(\mathbf{i d}_{k}\right)\left(a b c F_{2}-c y_{v} F_{2}\right) \\
& \quad-g\left(\mathbf{i d}_{k}\right)\left(a b c F_{2}-y_{v} c F_{2}\right)-h\left(\mathbf{i d}_{k}\right) c F_{2} \\
= & \alpha P_{2}+\left(w_{1}+c\right) \mathcal{H}_{2}\left(\mathbf{i d}_{k}\right)+r_{2} V_{2}-g\left(\mathbf{i d}_{k}\right) c d F_{2}-h\left(\mathbf{i d}_{k}\right) c F_{2} \\
= & \alpha P_{2}+\left(w_{1}+c\right) \mathcal{H}_{2}\left(\mathbf{i d}_{k}\right)+r_{2} V_{2}-c \mathcal{H}_{2}\left(\mathbf{i d}_{k}\right) \\
= & \alpha P_{2}+w_{1} \mathcal{H}_{2}\left(\mathbf{i d}_{k}\right)+r_{2} V_{2} .
\end{aligned}
$$

If $Z_{2}=(b c+\gamma) F_{2}$ the key will be partial semi-functional with $\gamma_{1}=\gamma, \pi=g\left(\mathbf{i d}_{k}\right)$ and $\pi_{j}=\lambda_{j}$ for $\ell+1 \leq j \leq h$. It is straightforward to check that $\mathcal{S} \mathcal{K}_{\mathbf{i d}_{k}}$ is a properly formed partial sf-key. Also, since $\left(\lambda_{j}\right)_{j \in[1, h]}, \nu$ are information theoretically hidden from the adversary, $\pi,\left(\pi_{j}\right)_{j \in[\ell+1, h]}$ are uniformly and independently distributed in $\mathscr{A}$ 's view.

$\mathscr{B}$ could attempt checking whether $\mathcal{S K}_{\mathbf{i d}_{k}}$ is semi-functional by creating a sf-ciphertext for $\mathbf{i d}_{k}$. Since $V_{1}^{\prime}=b F_{1}$ is not available to $\mathscr{B}$, the only way of doing this will lead to $\sigma$ being the same as $\pi$ (challenge ciphertext is created via this method). The ciphertext-key pair will be nominally semi-functional and thus provides no information to $\mathscr{B}$.

Challenge: $\mathscr{A}$ provides two message-identity pairs, $\left(M_{0}, \widehat{\mathbf{i d}}_{0}\right)$ and $\left(M_{1}, \widehat{\mathbf{i d}}_{1}\right)$ to $\mathscr{B}$. It chooses $\beta \in \in_{\mathrm{U}}\{0,1\}$, generates the challenge ciphertext as shown below.

$$
\begin{aligned}
& C_{0}=M_{\beta} \cdot e\left(d b x F_{1}, d F_{2}\right)^{\alpha} \\
& C_{1,1}=g\left(\widehat{\mathbf{i d}}_{\beta}\right)\left(d b x F_{1}\right)+h\left(\widehat{\mathbf{i d}}_{\beta}\right)\left(b x F_{1}\right) \\
& C_{1,2}=a g\left(\widehat{\mathbf{i d}}_{\beta}\right)\left(d b x F_{1}\right)+a h\left(\widehat{\mathbf{i d}}_{\beta}\right)\left(b x F_{1}\right)-g\left(\widehat{\mathbf{i d}}_{\beta}\right)\left(d^{2} x F_{1}\right) \\
& C_{1,3}=-y_{v} g\left(\widehat{\mathbf{i d}}_{\beta}\right)\left(d b x F_{1}\right)-h\left(\widehat{\mathbf{i d}}_{\beta}\right)\left(d b x F_{1}\right)-y_{v} h\left(\widehat{\mathbf{i d}}_{\beta}\right)\left(b x F_{1}\right) \\
& C_{2,1}=d b x F_{1}, C_{2,2}=a\left(d b x F_{1}\right)-d^{2} x F_{1}, C_{2,3}=-y_{v}\left(d b x F_{1}\right) .
\end{aligned}
$$

This sets $s=b x, \mu=-d^{2} x$ and $\sigma=g\left(\widehat{\mathbf{i d}}_{\beta}\right)$. Since $\lambda_{1}, \ldots, \lambda_{h}$ and $\nu$ are chosen uniformly at random from $\mathbb{Z}_{p}, \lambda_{1} X_{1}+\cdots+\lambda_{h} X_{h}+\nu$ is a pairwise independent function for variables $X_{1}, \ldots, X_{h}$ over $\mathbb{Z}_{p}$. As a result, $\pi=\lambda_{1} \mathrm{id}_{1}+\cdots+\lambda_{\ell} \mathrm{id}_{\ell}+\nu$ and $\sigma=\lambda_{1} \widehat{\hat{\mathrm{id}}}_{1}+\cdots+\lambda_{\hat{\ell}} \widehat{\mathrm{id}}_{\widehat{\ell}}+\nu$ are independent and uniformly distributed. $\mathscr{B}$ returns $\widehat{\mathcal{C}}=\left(C_{1, i}, C_{2, i}\right)_{i=1,2,3}$.

To show that $\widehat{\mathcal{C}}$ is indeed distributed properly, we show that $C_{1,3}$ is well-formed. Along the same lines, one can check the well-formedness of $C_{1,2}, C_{2,2}$ and $C_{2,3}$.

$$
\begin{aligned}
C_{1,3} & =-y_{v} g\left(\widehat{\mathbf{i d}}_{\beta}\right)\left(d b x F_{1}\right)-h\left(\widehat{\mathbf{i d}}_{\beta}\right)\left(d b x F_{1}\right)-y_{v} h\left(\widehat{\mathbf{i d}}_{\beta}\right)\left(b x F_{1}\right) \\
& =-y_{v} g\left(\widehat{\mathbf{i d}}_{\beta}\right)\left(d b x F_{1}\right)-y_{v} h\left(\widehat{\mathbf{i d}}_{\beta}\right)\left(b x F_{1}\right)-h\left(\widehat{\mathbf{i d}}_{\beta}\right)\left(d b x F_{1}\right)-g\left(\widehat{\mathbf{i d}}_{\beta}\right) d^{2} b x F_{1}+g\left(\widehat{\mathbf{i d}}_{\beta}\right) d^{2} b x F_{1} \\
& =-y_{v} b x \mathcal{H}_{1}\left(\widehat{\mathbf{i d}}_{\beta}\right)-d b x \mathcal{H}_{1}\left(\widehat{\mathbf{i d}}_{\beta}\right)+g\left(\widehat{\mathbf{i d}}_{\beta}\right) d^{2} x\left(b F_{1}\right) \\
& =-\tau \mathcal{H}_{1}\left(\widehat{\mathbf{i d}}_{\beta}\right)+\sigma \mu V_{1}^{\prime}
\end{aligned}
$$

Guess: $\mathscr{A}$ returns a bit $\beta^{\prime}$ as its guess for $\beta$.

When the instance is real, $\mathscr{B}$ simulates Game $_{k-1,1}$ and otherwise simulates Game ${ }_{k, 0} . \mathscr{B}$ returns 1 if $\mathscr{A}$ wins the game i.e., $\beta=\beta^{\prime}$; otherwise it returns 0 . Hence, $\mathscr{B}$ can solve the LW2 instance 
with advantage

$\operatorname{Adv}_{\mathcal{G}}^{\mathrm{LW} 2}(\mathscr{B})=\mid \operatorname{Pr}\left[\beta=\beta^{\prime} \mid Z_{2}\right.$ is real $]-\operatorname{Pr}\left[\beta=\beta^{\prime} \mid Z_{2}\right.$ is random $]|=| \operatorname{Pr}\left[X_{k-1,1}\right]-\operatorname{Pr}\left[X_{k, 0}\right] \mid$.

from which the statement of the lemma follows.

Lemma 3. $\left|\operatorname{Pr}\left[X_{k, 0}\right]-\operatorname{Pr}\left[X_{k, 1}\right]\right| \leq \varepsilon_{\mathrm{LW} 2}$ for $1 \leq k \leq q$.

The proof is reminiscent of Lemma 2. The reason is as follows: the structure of $\mathcal{S}_{2}$ is identical to $\mathcal{S}_{1}$ if the $\alpha P_{2}$ term is removed from $K_{2,1}$. Moreover, the simulator chooses $\alpha$ and creates $\alpha P_{2}$ independent of the instance. Hence the simulation will be similar except that the instance is now embedded in $\mathcal{S}_{2}$ and $\mathcal{S}_{1}$ is made semi-functional independent of the instance.

Lemma 4. $\left|\operatorname{Pr}\left[X_{q, 1}\right]-\operatorname{Pr}\left[X_{M \text {-rand }}\right]\right| \leq \varepsilon_{\mathrm{DBDH}-3}$.

Proof. $\mathscr{B}$ receives $\left(F_{1}, a F_{1}, b F_{1}, s F_{1}, F_{2}, a F_{2}, b F_{2}, s F_{2}, Z_{T}\right)$ as an instance of the DBDH-3 problem where $Z_{T}=e\left(F_{1}, F_{2}\right)^{a b s}$ (real) or $Z_{T} \in_{\mathrm{U}} \mathbb{G}_{T}$ (random).

Set-Up: With $y, v, v^{\prime}, y_{1}, \ldots, y_{h}, u$ chosen at random from $\mathbb{Z}_{p}, \mathscr{B}$ sets the parameters as

$$
\begin{aligned}
& P_{1}=y F_{1}, P_{2}=y F_{2}, a P_{1}=y\left(a F_{1}\right), V_{2}=v F_{2}, V_{2}^{\prime}=v^{\prime} F_{2}, \tau P_{1}=y v F_{1}+y v^{\prime}\left(a F_{1}\right) \\
& Q_{1, j}=y_{j} P_{1}=y_{j} y F_{1} \text { for } 1 \leq j \leq h, U_{1}=u P_{1}=u y F_{1}, e\left(P_{1}, P_{2}\right)^{\alpha}=e\left(a F_{1}, b F_{2}\right)^{y^{2}}
\end{aligned}
$$

implicitly setting $\alpha=a b$ and $\tau=v+a v^{\prime}$. The remaining parameters can be computed easily. $\mathscr{B}$ returns $\mathcal{P} \mathcal{P}$ to $\mathscr{A}$.

Phases 1 and 2: When $\mathscr{A}$ asks for the secret key for the $i$ 'th identity $\mathbf{i d}_{i}=\left(\operatorname{id}_{1}, \ldots, \mathrm{id}_{\ell}\right), \mathscr{B}$ chooses at random $w_{1}, w_{2}, r_{1}, r_{2}, r_{3}, r_{4},\left(z_{1, j}, z_{2, j}\right)_{j=1}^{h}$ and $\gamma_{1}^{\prime}, \gamma_{1}, \gamma_{2},\left(\pi_{j}\right)_{j=1}^{h}, \eta,\left(\eta_{j}\right)_{j=1}^{h}$ from $\mathbb{Z}_{p}$ and computes a semi-functional key for $\mathbf{i d}_{i}$ as follows.

$$
\begin{aligned}
& K_{1,1}=w_{1} P_{2}+r_{1} V_{2}-\gamma_{1}\left(a F_{2}\right), K_{1,2}=r_{1} V_{2}^{\prime}+\gamma_{1} F_{2}, K_{1,3}=r_{1} F_{2} \\
& K_{2,1}=\gamma_{1}^{\prime}\left(a F_{2}\right)+w_{1} h\left(\mathbf{i d}_{i}\right)\left(P_{2}\right)+r_{2} V_{2}, K_{2,2}=r_{2} V_{2}^{\prime}+y\left(b F_{2}\right)-\gamma_{1}^{\prime} F_{2}, K_{2,3}=r_{2} F_{2}, \\
& D_{j, 1}=w_{1} Q_{2, j}+z_{1, j} V_{2}-\gamma_{1} \pi_{j}\left(a F_{2}\right), D_{j, 2}=z_{1, j} V_{2}^{\prime}+\gamma_{1} \pi_{j} F_{2}, D_{j, 3}=z_{1, j} F_{2} \text { for } \ell+1 \leq j \leq h . \\
& J_{1,1}=w_{2} P_{2}+r_{3} V_{2}-\gamma_{2}\left(a F_{2}\right), J_{1,2}=r_{3} V_{2}^{\prime}+\gamma_{2} F_{2}, J_{1,3}=r_{3} F_{2} \\
& J_{2,1}=w_{2} h\left(\mathbf{i d}_{i}\right)\left(P_{2}\right)+r_{4} V_{2}-\gamma_{2} \eta\left(a F_{2}\right), J_{2,2}=r_{4} V_{2}^{\prime}+\gamma_{2} \eta F_{2}, J_{2,3}=r_{4} F_{2}, \\
& E_{j, 1}=w_{2} Q_{2, j}+z_{2, j} V_{2}-\gamma_{2} \eta_{j}\left(a F_{2}\right), E_{j, 2}=z_{2, j} V_{2}^{\prime}+\gamma \eta_{j} F_{2}, E_{j, 3}=z_{2, j} F_{2} \text { for } \ell+1 \leq j \leq h .
\end{aligned}
$$

Here the relation $a \gamma_{1}^{\prime}=b y-\gamma_{1} \pi$ is implicitly set by the simulator. Calculations provided below justify that $K_{2,1}$ and $K_{2,2}$ have the correct distribution. Other elements have the correct form and distribution.

$$
\begin{aligned}
K_{2,1} & =\gamma_{1}^{\prime}\left(a F_{2}\right)+w_{1} h\left(\mathbf{i d}_{i}\right)\left(P_{2}\right)+r_{2} V_{2} & K_{2,2} & =r_{2} V_{2}^{\prime}+y\left(b F_{2}\right)-\gamma_{1}^{\prime} F_{2} \\
& =\left(b y-\gamma_{1} \pi\right)\left(a F_{2}\right)+w_{1} h\left(\mathbf{i d}_{i}\right)\left(P_{2}\right)+r_{2} V_{2} & & =r_{2} V_{2}^{\prime}+y\left(b F_{2}\right)-\left(b y-\gamma_{1} \pi\right) F_{2} \\
& =a b\left(y F_{2}\right)+w_{1} h\left(\mathbf{i d}_{i}\right)\left(P_{2}\right)+r_{2} V_{2}-a \gamma_{1} \pi F_{2} & & =r_{2} V_{2}^{\prime}+y\left(b F_{2}\right)-b y F_{2}+\gamma_{1} \pi F_{2} \\
& =\alpha P_{2}+w_{1} h\left(\mathbf{i d}_{i}\right)\left(P_{2}\right)+r_{2} V_{2}-a \gamma_{1} \pi F_{2} . & & =r_{2} V_{2}^{\prime}+\gamma_{1} \pi F_{2} .
\end{aligned}
$$

Observe that $\mathscr{B}$ does not know $\alpha$ or $\alpha F_{2}$ and hence cannot create a normal key.

Challenge: $\mathscr{B}$ receives two pairs $\left(M_{0}, \widehat{\mathbf{i d}}_{0}\right)$ and $\left(M_{1}, \widehat{\mathbf{i d}}_{1}\right)$ from $\mathscr{A}$. It samples $\beta \longleftarrow$ U $\{0,1\}$, $\mu^{\prime} \stackrel{\mathrm{U}}{\longleftarrow} \mathbb{Z}_{p}$ and generates a semi-functional challenge ciphertext as follows.

$$
\begin{aligned}
& C_{0}=M_{\beta} \times Z_{T} \\
& C_{1,1}=y h\left(\widehat{\mathbf{i d}}_{\beta}\right) s F_{1}, C_{1,2}=h\left(\widehat{\mathbf{i d}}_{\beta}\right) \mu^{\prime} F_{1}, C_{1,3}=-v y h\left(\widehat{\mathbf{i d}}_{\beta}\right)\left(s F_{1}\right)-v^{\prime} h\left(\widehat{\mathbf{i d}}_{\beta}\right) \mu^{\prime} F_{1} \\
& C_{2,1}=y\left(s F_{1}\right), C_{2,2}=\mu^{\prime} F_{1}, C_{2,3}=-y v\left(s F_{1}\right)-v^{\prime} \mu^{\prime} F_{1}
\end{aligned}
$$


with $\mu^{\prime}=a s y+\mu$ and $\sigma=h\left(\widehat{\mathbf{i d}}_{\beta}\right)$. The challenge ciphertext $\widehat{\mathcal{C}}=\left(C_{0}, C_{1,1}, C_{1,2}, C_{1,3}, C_{2,1}, C_{2,2}, C_{2,3}\right)$ is returned to $\mathscr{A}$.

Guess: $\mathscr{A}$ returns its guess $\beta^{\prime}$ of $\beta$.

It is clear that $\widehat{\mathcal{C}}$ is a semi-functional encryption of $M_{\beta}$ when $Z_{T}=e\left(P_{1}, P_{2}\right)^{a b s}$. And when $Z_{T} \in \in_{\mathrm{U}} \mathbb{G}_{T} \widehat{\mathcal{C}}$ would be a semi-functional encryption of a random message. Hence $\mathscr{B}$ simulates Game $_{q, 1}$ or Game final $_{\text {according to }} Z_{T}$ being real or random respectively. If the algorithm $\mathscr{B}$ returns 1 when $\beta=\beta^{\prime}$ and 0 otherwise, it can solve the DBDH-3 instance with advantage

$$
\operatorname{Adv}_{\mathcal{G}}^{\mathrm{DBDH}-3}(\mathscr{B})=\mid \operatorname{Pr}\left[\beta=\beta^{\prime} \mid Z_{T} \text { is real }\right]-\operatorname{Pr}\left[\beta=\beta^{\prime} \mid Z_{T} \text { is random }\right]|=| \operatorname{Pr}\left[X_{q, 1}\right]-\operatorname{Pr}\left[X_{\text {final }}\right] \mid .
$$

Lemma 5. $\left|\operatorname{Pr}\left[X_{M-r a n d}\right]-\operatorname{Pr}\left[X_{\text {final }}\right]\right| \leq \varepsilon_{\mathrm{A} 1}$.

Proof. Let $\left(\mathcal{G}, F_{1}, z F_{1}, d z F_{1}, a z F_{1}, a d z F_{1}, s z F_{1}, F_{2}, z F_{2}, a F_{2}, x F_{2},(d z-a x) F_{2}, Z_{1}\right)$ be the instance of $\mathrm{A} 1$ provided to $\mathscr{B}$. Let $Z_{1}=c \cdot s d z F_{1} . \mathscr{B}$ has to determine whether $c=1$ or $c \in_{\mathrm{U}} \mathbb{Z}_{p}$. The game is simulated as follows.

Set-Up: Pick $\alpha, v, v^{\prime}, y_{1}, \ldots, y_{h}, u \stackrel{\mathrm{U}}{\longleftarrow} \mathbb{Z}_{p}$ and set the parameters as

$$
\begin{gathered}
P_{1}=z F_{1}, V_{2}=v F_{2}, V_{2}^{\prime}=v^{\prime} F_{2}, Q_{1, j}=y_{j}\left(d z F_{1}\right), U_{1}=u\left(d z F_{1}\right), \\
a P_{1}=a z P_{1}, a Q_{1, j}=y_{j}\left(a d z F_{1}\right), a U_{1}=u\left(a d z F_{1}\right),
\end{gathered}
$$

where $j=1, \ldots, h$ and similarly the elements $\tau P_{1}, \tau Q_{1, j}$ and $\tau U_{1}$. Compute $e\left(P_{1}, P_{2}\right)^{\alpha}=$ $e\left(z F_{1}, z F_{2}\right)^{\alpha} . \mathscr{B}$ returns $\mathcal{P} \mathcal{P}$ to $\mathscr{A} . \mathscr{B}$ knows $P_{2}=z F_{2}$ and $\alpha$ but not $Q_{2, j}$ 's and $U_{2}$. The main idea is to mask the components required to create identity-hash in $\mathbb{G}_{2}$ by a scalar multiple of $a F_{2}$ so that only semi-functional keys can be created.

Key Extraction Phases 1 and 2: $\mathscr{B}$ picks $w_{1}, w_{2}, r_{1}, r_{2}, r_{3}, r_{4},\left(z_{1, j}, z_{2, j}\right)_{j=1}^{h} \stackrel{\mathrm{U}}{\longleftarrow} \mathbb{Z}_{p}, \gamma_{1}, \gamma_{2} \stackrel{\mathrm{U}}{\longleftarrow}$ $\mathbb{Z}_{p}^{\times}$and $\pi^{\prime},\left(\pi_{j}^{\prime}\right)_{j=1}^{h}, \eta^{\prime},\left(\eta_{j}^{\prime}\right)_{j=1}^{h} \stackrel{\mathrm{U}}{\longleftarrow} \mathbb{Z}_{p}$. It then computes the key for the $i$-th identity vector id $\mathbf{i d}_{i}=$ $\left(\mathrm{id}_{1}, \ldots, \mathrm{id}_{\ell}\right)$ as follows.

$$
\begin{aligned}
& K_{1,1}=w_{1}\left(z F_{2}\right)+r_{1} V_{2}-\gamma_{1} a F_{2}, K_{1,2}=r_{1} V_{2}^{\prime}+\gamma_{1} F_{2}, K_{1,3}=r_{1} F_{2} \\
& K_{2,1}=\alpha z F_{2}+w_{1} h\left(\mathbf{i d}_{i}\right)(d z-a x) F_{2}+r_{2} V_{2}-\gamma_{1} \pi^{\prime}\left(a F_{2}\right), K_{2,2}=r_{2} V_{2}^{\prime}+w_{1} h\left(\mathbf{i d}_{i}\right) x F_{2}+\gamma_{1} \pi^{\prime} F_{2}, K_{2,3}=r_{2} F_{2}, \\
& J_{1,1}=w_{2}\left(z F_{2}\right)+r_{3} V_{2}-\gamma_{2} a F_{2}, J_{1,2}=r_{3} V_{2}^{\prime}+\gamma_{2} F_{2}, J_{1,3}=r_{3} F_{2} \\
& J_{2,1}=w_{2} h\left(\mathbf{i d}_{i}\right)(d z-a x) F_{2}+r_{4} V_{2}-\gamma_{2} \eta^{\prime}\left(a F_{2}\right), J_{2,2}=r_{4} V_{2}^{\prime}+w_{2} h\left(\mathbf{i d}_{i}\right) x F_{2}+\gamma_{2} \eta^{\prime} F_{2}, J_{2,3}=r_{4} F_{2},
\end{aligned}
$$

For $\ell+1 \leq j \leq h$,

$D_{j, 1}=w_{1} y_{j}(d z-a x) F_{2}+z_{1, j} V_{2}-\gamma_{1} \pi_{j}^{\prime}\left(a F_{2}\right), D_{j, 2}=z_{1, j} V_{2}^{\prime}+w_{1} y_{j}\left(x F_{2}\right)+\gamma_{1} \pi_{j}^{\prime} F_{2}, D_{j, 3}=z_{1, j} F_{2}$

$E_{j, 1}=w_{2} y_{j}(d z-a x) F_{2}+z_{2, j} V_{2}-\gamma_{2} \eta_{j}^{\prime}\left(a F_{2}\right), E_{j, 2}=z_{2, j} V_{2}^{\prime}+w_{2} y_{j}\left(x F_{2}\right)+\gamma_{2} \eta_{j}^{\prime} F_{2}, E_{j, 3}=z_{2, j} F_{2}$

setting $\pi=\pi^{\prime}+\gamma_{1}^{-1} w_{1} h\left(\mathbf{i d}_{i}\right) x, \pi_{j}=\pi_{j}^{\prime}+\gamma_{1}^{-1} w_{1} y_{j} x, \eta=\eta^{\prime}+\gamma_{2}^{-1} w_{2} h\left(\mathbf{i d}_{i}\right) x$ and $\eta_{j}=\eta_{j}^{\prime}+\gamma_{2}^{-1} w_{2} y_{j} x$. Since all these scalars are additively randomised they remain properly distributed in the adversary's 
view. We show that $D_{j, 1}, D_{j, 2}$ are well-formed; the rest can be verified in a similar fashion.

$$
\begin{aligned}
D_{j, 1} & =w_{1} y_{j}(d z-a x) F_{2}+z_{1, j} V_{2}-\gamma_{1} \pi_{j}^{\prime}\left(a F_{2}\right) \\
& =w_{1} y_{j} d z F_{2}-w_{1} y_{j} a x F_{2}+z_{1, j} V_{2}-\gamma_{1}\left(\pi_{j}-\gamma_{1}^{-1} w_{1} y_{j} x\right)\left(a F_{2}\right) \\
& =w_{1} y_{j} d z F_{2}-w_{1} y_{j} a x F_{2}+z_{1, j} V_{2}-a \gamma_{1} \pi_{j} F_{2}+w_{1} y_{j} a x F_{2} \\
& =w_{1} y_{j} d z F_{2}+z_{1, j} V_{2}-a \gamma_{1} \pi_{j} F_{2} \\
D_{j, 2} & =z_{1, j} V_{2}^{\prime}+w_{1} y_{j}\left(x F_{2}\right)+\gamma_{1} \pi_{j}^{\prime} F_{2} \\
& =z_{1, j} V_{2}^{\prime}+w_{1} y_{j}\left(x F_{2}\right)+\gamma_{1}\left(\pi_{j}-\gamma_{1}^{-1} w_{1} y_{j} x\right) F_{2} \\
& =z_{1, j} V_{2}^{\prime}+w_{1} y_{j} x F_{2}+\gamma_{1} \pi_{j} F_{2}-w_{1} y_{j} x F_{2} \\
& =z_{1, j} V_{2}^{\prime}+\gamma_{1} \pi_{j} F_{2}
\end{aligned}
$$

Challenge: $\mathscr{B}$ receives two pairs of messages and identity vectors $\left(M_{0}, \widehat{\mathbf{i d}}_{0}\right)$ and $\left(M_{1}, \widehat{\mathbf{i d}}_{1}\right)$ from $\mathscr{A}$. It chooses $\beta \stackrel{\mathrm{U}}{\longleftarrow}\{0,1\}$ and $a^{\prime}, \xi \stackrel{\mathrm{U}}{\longleftarrow} \mathbb{Z}_{p}$ at random and generates a semi-functional challenge ciphertext as follows.

$$
\begin{aligned}
& C_{0} \stackrel{\mathrm{U}}{\longleftarrow} \mathbb{G}_{T} \\
& C_{1,1}=h\left(\widehat{\mathbf{i d}}_{\beta}\right) Z_{1}, C_{1,2}=a^{\prime} h\left(\widehat{\mathbf{i d}}_{\beta}\right) Z_{1}+\xi F_{1}, C_{1,3}=-v h\left(\widehat{\mathbf{i d}}_{\beta}\right) Z_{1}-v^{\prime} a^{\prime} h\left(\widehat{\mathbf{i d}}_{\beta}\right) Z_{1}-v^{\prime} \xi F_{1}, \\
& C_{2,1}=s z F_{1}, C_{2,2}=a^{\prime} s z F_{1}, C_{2,3}=-v\left(s z F_{1}\right)-v^{\prime} a^{\prime}\left(s z F_{1}\right),
\end{aligned}
$$

where $a^{\prime}=a+\mu^{\prime}, \mu=\mu^{\prime} s z$ and $\xi=\mu \sigma^{\prime}$. The challenge ciphertext $\widehat{\mathcal{C}}=$ $\left(C_{0}, C_{1,1}, C_{1,2}, C_{1,3}, C_{2,1}, C_{2,2}, C_{2,3}\right)$ is returned to $\mathscr{A}$. The computations below illustrate that $\widehat{\mathcal{C}}$ is a semi-functional encryption with $\sigma=\sigma^{\prime}+c d h\left(\widehat{\mathbf{i d}}_{\beta}\right)$.

$$
\begin{aligned}
C_{1,2} & =a^{\prime} h\left(\widehat{\mathbf{i d}}_{\beta}\right) Z_{1}+\xi F_{1} \\
& =\left(a+\mu^{\prime}\right) h\left(\widehat{\mathbf{i d}}_{\beta}\right) c s d z F_{1}+\mu \sigma^{\prime} F_{1} \\
& =a h\left(\widehat{\mathbf{i d}}_{\beta}\right) c s d z F_{1}+\mu^{\prime} h\left(\widehat{\mathbf{i d}}_{\beta}\right) c s d z F_{1}+\mu \sigma^{\prime} F_{1} \\
& =a s \mathcal{H}_{1}\left(\widehat{\mathbf{i d}}_{\beta}\right)+\left(\mu^{\prime} s z\right)\left(c d h\left(\widehat{\mathbf{i d}}_{\beta}\right)\right) F_{1}+\mu \sigma^{\prime} F_{1} \\
& =a s \mathcal{H}_{1}\left(\widehat{\mathbf{i d}}_{\beta}\right)+\mu\left(c d h\left(\widehat{\mathbf{i d}}_{\beta}\right)\right) F_{1}+\mu \sigma^{\prime} F_{1} \\
& =a s \mathcal{H}_{1}\left(\widehat{\mathbf{i d}}_{\beta}\right)+\mu \sigma F_{1}
\end{aligned}
$$

Observe that $C_{1,1}=s \mathcal{H}_{1}\left(\widehat{\mathbf{i d}}_{\beta}\right)=\left(c \cdot h\left(\widehat{\mathbf{i d}}_{\beta}\right)\right)\left(s d z F_{1}\right)$. If $c=1$, then $\sigma=\sigma^{\prime}+d h\left(\widehat{\mathbf{i d}}_{\beta}\right)$ and $\widehat{\mathcal{C}}$ is encrypted under $\widehat{\mathbf{i d}}_{\beta}$. Otherwise, $c$ is random, causing $h\left(\widehat{\mathbf{i d}}_{\beta}\right)$ and consequently the target identity and $\sigma$ to be random quantities.

Guess: $\mathscr{A}$ returns its guess $\beta^{\prime}$ of $\beta$.

If the algorithm $\mathscr{B}$ returns 1 when $\beta=\beta^{\prime}$ and 0 otherwise, it can solve the A1 instance with advantage

$$
\operatorname{Adv}_{\mathcal{G}}^{\mathrm{A} 1}(\mathscr{B})=\mid \operatorname{Pr}\left[\beta=\beta^{\prime} \mid Z_{1} \text { is real }\right]-\operatorname{Pr}\left[\beta=\beta^{\prime} \mid Z_{1} \text { is random }\right]|=| \operatorname{Pr}\left[X_{M \text {-rand }}\right]-\operatorname{Pr}\left[X_{\text {final }}\right] \mid .
$$

\section{Conclusion}

We have extended the Lewko-Waters IBE scheme using asymmetric pairings to a constant-size ciphertext HIBE. In addition to CPA-security the HIBE scheme possesses anonymity. Security of 
them HIBE is based on the assumptions LW1, LW2, DBDH-3 and a new assumption A1 that we introduce. This HIBE is the first example of an anonymous, adaptive-id secure, constant-size ciphertext HIBE which can be instantiated using Type-3 pairings. The assumptions used are static but non-standard. It would be interesting to explore constructions employing dual pairing vector spaces to obtain security under standard assumptions.

\section{References}

1. Michel Abdalla, Mihir Bellare, Dario Catalano, Eike Kiltz, Tadayoshi Kohno, Tanja Lange, John Malone-Lee, Gregory Neven, Pascal Paillier, and Haixia Shi. Searchable encryption revisited: Consistency properties, relation to anonymous IBE, and extensions. In Victor Shoup, editor, CRYPTO, volume 3621 of Lecture Notes in Computer Science, pages 205-222. Springer, 2005.

2. Dan Boneh, Xavier Boyen, and Eu-Jin Goh. Hierarchical identity-based encryption with constant size ciphertext. In Ronald Cramer, editor, EUROCRYPT, volume 3494 of Lecture Notes in Computer Science, pages 440-456. Springer, 2005. Full version available at Cryptology ePrint Archive; Report 2005/015.

3. Dan Boneh and Matthew K. Franklin. Identity-based encryption from the Weil pairing. SIAM J. Comput., 32(3):586-615, 2003. Earlier version appeared in the proceedings of CRYPTO 2001.

4. Xavier Boyen and Brent Waters. Anonymous hierarchical identity-based encryption (without random oracles). In Cynthia Dwork, editor, CRYPTO, volume 4117 of Lecture Notes in Computer Science, pages 290-307. Springer, 2006.

5. Sanjit Chatterjee and Alfred Menezes. On cryptographic protocols employing asymmetric pairings - the role of $\psi$ revisited. Discrete Applied Mathematics, 159(13):1311-1322, 2011.

6. Clifford Cocks. An identity-based encryption scheme based on quadratic residues. In Bahram Honary, editor, IMA Int. Conf., volume 2260 of Lecture Notes in Computer Science, pages 360-363. Springer, 2001.

7. Angelo De Caro, Vincenzo Iovino, and Giuseppe Persiano. Fully secure anonymous hibe and secret-key anonymous ibe with short ciphertexts. In Marc Joye, Atsuko Miyaji, and Akira Otsuka, editors, Pairing-Based Cryptography - Pairing 2010, volume 6487 of Lecture Notes in Computer Science, pages 347-366. Springer Berlin / Heidelberg, 2010.

8. Léo Ducas. Anonymity from asymmetry: New constructions for anonymous hibe. In Josef Pieprzyk, editor, CT-RSA, volume 5985 of Lecture Notes in Computer Science, pages 148-164. Springer, 2010.

9. Steven D. Galbraith, Kenneth G. Paterson, and Nigel P. Smart. Pairings for cryptographers. Discrete Applied Mathematics, 156(16):3113-3121, 2008.

10. Craig Gentry and Alice Silverberg. Hierarchical ID-based cryptography. In Yuliang Zheng, editor, ASIACRYPT, volume 2501 of Lecture Notes in Computer Science, pages 548-566. Springer, 2002.

11. Jeremy Horwitz and Ben Lynn. Toward hierarchical identity-based encryption. In Lars R. Knudsen, editor, EUROCRYPT, volume 2332 of Lecture Notes in Computer Science, pages 466-481. Springer, 2002.

12. Allison B. Lewko and Brent Waters. New techniques for dual system encryption and fully secure HIBE with short ciphertexts. In Daniele Micciancio, editor, TCC, volume 5978 of Lecture Notes in Computer Science, pages 455-479. Springer, 2010.

13. Jong Hwan Park and Dong Hoon Lee. Anonymous hibe: Compact construction over prime-order groups. IEEE Transactions on Information Theory, 59(4):2531-2541, 2013.

14. Jae Hong Seo, Tetsutaro Kobayashi, Miyako Ohkubo, and Koutarou Suzuki. Anonymous hierarchical identitybased encryption with constant size ciphertexts. In Stanislaw Jarecki and Gene Tsudik, editors, Public Key Cryptography, volume 5443 of Lecture Notes in Computer Science, pages 215-234. Springer, 2009.

15. Adi Shamir. Identity-based cryptosystems and signature schemes. In G. R. Blakley and David Chaum, editors, CRYPTO, volume 196 of Lecture Notes in Computer Science, pages 47-53. Springer, 1984.

16. Nigel P. Smart and Frederik Vercauteren. On computable isomorphisms in efficient asymmetric pairing-based systems. Discrete Applied Mathematics, 155(4):538-547, 2007.

17. Brent Waters. Dual system encryption: Realizing fully secure IBE and HIBE under simple assumptions. In Shai Halevi, editor, CRYPTO, volume 5677 of Lecture Notes in Computer Science, pages 619-636. Springer, 2009. 